\title{
Some Advances on Antagonist Effects of Grain Boundaries between the Trapping Process and the Fast Diffusion Path Investigated on Nickel Bicrystals
}

\author{
xavier FEAUGAS ( $\nabla$ xfeaugas@univ-Ir.fr) \\ University of La Rochelle \\ jiaqi Li \\ University of La Rochelle \\ Malek Hallil \\ University of La Rochelle \\ Arnaud Metsue \\ University of La Rochelle \\ Abdelali Oudriss \\ University of La Rochelle \\ Jamaa Bouhattate \\ University of La Rochelle
}

\section{Research Article}

Keywords: Hydrogen-grain-boundaries, intergranular fracture, segregation, trapping and diffusion

Posted Date: May 21st, 2021

DOl: https://doi.org/10.21203/rs.3.rs-461172/v1

License: (c) (1) This work is licensed under a Creative Commons Attribution 4.0 International License.

Read Full License

Version of Record: A version of this preprint was published at Scientific Reports on July 30th, 2021. See the published version at https://doi.org/10.1038/s41598-021-94107-6. 


\title{
Some advances on antagonist effects of grain boundaries between the trapping process and the fast diffusion path investigated on nickel bicrystals
}

\author{
J. Li, A. Hallil, A. Metsue, A. Oudriss, J. Bouhattate, X. Feaugas \\ LaSIE UMR CNRS 7356, La Rochelle Université, Av. Michel Crépeau, 17042 La Rochelle Cedex 1, \\ France.
}

\begin{abstract}
Hydrogen-grain-boundaries interactions and their role in intergranular fracture are well accepted as one of the key features in understanding hydrogen embrittlement in a large variety of common engineer situations. These interactions implicate some fundamental processes classified as segregation, trapping and diffusion of the solute which can be studied as a function of grain boundary configuration. In the present study, we carried out an extensive analysis of four grainboundaries based on the complementary of atomistic calculations and experimental data. We demonstrate that elastic deformation has an important contribution on the segregation energy which cannot be simply reduced to a volume change and need to consider the deviatoric part of strain. Additionally, some significant configurations of the segregation energy depend on the long-range elastic distortion and allows to rationalize the elastic contribution in three terms. By investigating the different energy barriers involved to reach all the segregation sites, the antagonist impact of grain boundaries on hydrogen diffusion and trapping process was elucidated. The segregation energy and migration energy are two fundamental parameters in order to classify the grain-boundaries as a trapping location or short circuit for diffusion.
\end{abstract}




\section{Introduction}

Hydrogen has a strong tendency to segregate or interact with structural defects as point defects, solid solution species, precipitates, dislocations and internal interfaces (inter-phases, grainboundaries, ...). This situation affects the apparent solubility and the mobility of hydrogen and consequently modifies the embrittlement process of metals and their alloys ${ }^{1}$. Many studies ${ }^{2-}$ ${ }^{6}$ support the role of hydrogen state in the control of the properties of hydrogenated materials in a large diversity of cases (hydrogen diffusion, hydrogen induced cracking, electrical properties in semiconductors, catalyze ...) and more precisely their mechanical behavior (embrittlement itself). In this context, hydrogen seems to have a larger implication on intergranular fracture ${ }^{7-13}$. While segregation and diffusion of hydrogen at grain boundaries (GBs) have been of great interest for understanding hydrogen embrittlement (HE), the difficulty of hydrogen analysis at grain boundary scale is the limited number of studies carried out in this subject for nickel alloys (see for a review on the subject references ${ }^{14,15}$ ). The interactions of hydrogen with grains and grain boundaries are often treated numerically and experimentally so as to separate with a little confrontation $^{16-18}$. Also, at the atomistic scale, there are many works that are rapidly interested in inter-granular decohesion ${ }^{19-23}$ in relation to segregation without a precise focus on the elementary process which occurs at the grain boundary. In contrast, the short circuit of diffusion within the grain boundaries remains a remarkable complex subject that would require more in-depth analysis.

There is little available experimental data of the hydrogen segregation for properly defined GBs. Indeed, adequately characterizing a GB persists to be an engineering challenge, especially the crystallographic orientation and the misorientation angle need to be suitably controlled. For two decades, a large debate was supported by many studies on the possible conflict between the fact that grain-boundaries can be a either a trapping site and/or a short circuit of diffusion ${ }^{24-28}$ in fcc materials. More recently, based on a larger experimental investigation Oudriss et al. ${ }^{27-29}$ have reported that the GBs with low misorientation $(\Sigma 1)$ and a category of "special" grain boundaries $(\Sigma 3-\Sigma 29)$ are usually preferential areas for hydrogen trapping in polycrystalline nickel. In fact, considering their ordered structure, this kind of boundary is accommodated by defects (dislocations, vacancies and more complex organization) that represent potential traps of hydrogen. In opposite, the high angle "random" grain boundaries are considered as the "disordered phase" where the hydrogen diffusion is accelerated in relation to an eased path associated with lower energy barrier. The predominance of one phenomenon over the other depends on the grain boundary energy and the excess of free volume ${ }^{28}$. These results gathered from a correlation between large data of diffusion coefficient and grain boundary character seems to highlight some exceptions ${ }^{29}$ which suggest a considerable diversity of local processes. These mechanisms were improved and discussed an extensive variety of experimental technics with a 
high spatial resolution which has been reviewed recently ${ }^{14,15}$. In fcc metals and alloys, the existence of short-circuit diffusion paths of hydrogen was illustrated using the hydrogen microprint technics and Secondary Ion Mass Spectrometry (SIMS) mapping ${ }^{14,15,29-32}$. The preferential ingress of hydrogen along grain boundaries was observed by Tanaka et al. ${ }^{32}$ using Ga-FIB-TOF-SIMS to directly visualize deuterium distribution in fcc steel. Microprint technic shows that not all grain boundaries are generally decorated with Ag crystals, which suggest that hydrogen transport capacity of a boundary depends on its microstructural specificity (character, orientation, ... $)^{30,31}$. More recently, Tof-SIMS and EBSD were combined to investigate statistically hydrogen distribution around grain boundaries in polycrystalline nickel ${ }^{29}$. Our results suggest that grain boundaries can be categorized into two families according to how hydrogen is distributed across the grain boundary. The first family designates random grain boundaries which reveal a sharp gap for hydrogen concentration profile across the grain boundaries. The second one is special $\Sigma 3^{\mathrm{n}}$ grain boundaries which presents a smooth gradient of hydrogen concentration cross the grain boundary. Despite these new relevant results, it is clear to conclude that actually it stays difficult to demonstrate that hydrogen distribution results in a heterogeneous behavior of diffusion and segregation processes or both. Recent in situ SKPFM analyses using for detecting the local hydrogen distribution around GBs, demonstrate that a random GB with a misorientation of $43^{\circ}$ does not significantly facilitate hydrogen diffusion, while a coherent $\Sigma 3$ twin GB provides a fast path for hydrogen transport ${ }^{33}$. This last result seems in opposite with Oudriss works ${ }^{28-29}$ and questions the simple view based on random and Coincidence Site Lattice character (CSL $-\Sigma)$. Additionally, SIMS mapping ${ }^{29,34-36}$ and recent Atom Probe Tomography observations ${ }^{37}$ highlight a gradient of hydrogen content with a path length higher than the GBs thickness which suggests that hydrogen diffusivity and segregation processes cannot be only discussed in relation to the local structure of grain boundaries.

Based on atomistic simulations, several computational efforts have focused on the hydrogen segregation and diffusion properties and embrittlement consequences for some selected grain boundary in nicke ${ }^{38-58}$. Classically, the grain boundaries are characterized by their energy, excess volume and geometric parameters such as Coincidence Site Lattice; easily accessible data using density functional theory (DFT) or molecular dynamics (MD) simulations ${ }^{59,60}$. These characteristics are determining factors on the interaction properties of solutes with the GB; however such global values sometimes appear to be far from representative of local behaviour. According to extensive atomistic simulations, the segregation energy is essential to the understanding of dynamic processes of solute evolution in materials. The minimum segregation energy, commonly used to characterize GB capacity to interact with hydrogen, vary significantly from - 0.04 to $-0.37 \mathrm{eV}$ depending on the GB character ${ }^{40,46,47,49-53,55}$. The effects of last one can be evaluated on the base of literature data for $\Sigma 3(111)(-0.04 \mathrm{eV})^{46,47}, \Sigma 3(110)(-0.21$ 
$\mathrm{eV})^{47}, \Sigma 3(221)(-0.21 \mathrm{eV})^{47}, \Sigma 3(112)(-0.24 \mathrm{eV})^{47}, \Sigma 5(012)(-0.23 \text { to }-0.37 \mathrm{eV})^{40,46,49-52}, \Sigma 5(001)$ $(-0.16 \mathrm{eV})^{53}, \Sigma 5(310)(-0.32 \mathrm{eV})^{55}, \Sigma 9(221)(-0.2 \mathrm{eV})^{47}, \Sigma 99(557)(-0.15 \mathrm{eV})^{47}$ and $\Sigma 17(140)(-$ $0.34 \mathrm{eV})^{55}$. These values can be significantly modified as a function of the conditions applied to atomistic calculations (size of the box, DFT or EAM potential ...) but globally the minimum segregation energy increases with the increases of the CSL index. Hallil et al. suggest that for $\Sigma 3$ GBs, that the GB character (energy, and excess volume) can be treated by the notion of the inclination angle $\phi$ between the two symmetrical tilt grain boundaries (STGB): coherent twin boundary (CTB) and symmetrical incoherent twin boundary (SITB) configurations ${ }^{47,61}$. Energy and excess volume expands with $\phi$ and at the same time the minimum segregation energy of hydrogen grows ${ }^{47}$. Based on $\mathrm{MD} / \mathrm{MC}$ simulations, larger systems can be investigated. On the other hand, Moody et al. ${ }^{48}$ have pointed out that the hydrogen concentration is enhanced in tilt $\Sigma 9(221)$ high energy grain boundary in nickel. More recently, Brien and Foilles ${ }^{44}$ have studied the hydrogen segregation in inclined $\Sigma 3<110>$ nickel GBs using the hybrid MC/MD and an analytic segregation model. The maximum concentration of hydrogen occurs at the boundary at the inclination with the highest enthalpy. This result also gives a correlation between the hydrogen segregation and the GBs energy since the GB energy amplifies with the inclination angle for the nickel GB $\Sigma 3<110>$. The hydrogen segregation phenomenon is more pronounced for high energy GBs which may be explained by the high excess volume for these GBs. All these outcomes suggest a correlation with the geometric and energetic configuration of grain boundaries and segregation properties, but the physical bases of this relationship stay ambiguous. More recently, the local state was considered in some nickel grain-boundaries ${ }^{47,57}$. Some correlation seems to be possible between the local deformation of hydrogen segregation volume defined by polyhedrons using the Voronoi tessellation method. These studies suggest that elastic dilatation and distortion deformation of the site is partially responsible for the segregation energy. Based on these considerations, some authors have used a continuum approach to evaluate the impact of the elastic field associated with GBs on segregation processes ${ }^{62}$. The respective contribution of short and long-range stress continues to be an open question.

The roles of grain boundaries (GBs) in hydrogen diffusion processes were determined from density functional theory calculations by some authors in fcc metals ${ }^{52,63,64}$. The energy barriers along the diffusion path towards and within GBs has been related for $\Sigma 3<110>\{111\}$ and $\Sigma 11<110>\{113)$ in fcc Fe- $\gamma^{63}$, and for $\Sigma 3<110>\{111\}^{52,64}, \Sigma 5<100>\{210\}^{52,64}, \Sigma 5<100>\{310\}^{64}$, $\Sigma 11<110>\{113\}^{64}, \Sigma 25<100>\{430\}^{64}$ and $\Sigma 41<100>\{540\}^{64}$ in fcc Nickel. In fcc Fe- $\gamma$, the $\Sigma 3$ GB repels hydrogen and the $\Sigma 11$ offers an easy diffusion path parallel to the GB plane ${ }^{63}$. In fcc nickel, the $\Sigma 3$ and $\Sigma 11$ present a quite similar diffusion behavior tho the bulk ${ }^{64}$ and $\Sigma 5$ GBs exhibit lowbarrier paths to facilitate hydrogen diffusion along the $\mathrm{GBs}^{52,64}$ while $\Sigma 25$ and $\Sigma 41$ exhibit highbarrier regions which suggest a slower diffusion of hydrogen than the bulk ${ }^{64}$. The authors suggest 
that a trapping model in relation with the dislocation density is sufficient to relate these data ${ }^{64}$. Despite these appreciable results, a minor confrontation was proposed in the literature between hydrogen diffusivity and segregation capability of GBs which doesn't offer the opportunity to clarify the trapping process inside the GBs.

Despite numerous experimental and numerical studies, short-circuits of diffusion and trapping processes within grain boundaries in fcc metals and alloys remain a complex subject that is still poorly understood. Furthermore, the confrontation of experience and numerical works has not been currently used in this subject which reduces the quality of the interpretations.

In the present work, a substantial effort was made to gain further understanding of the key issues of hydrogen segregation and diffusion processes near GBs. The hydrogen/grain-boundaries interactions have been examined for four different configurations of nickel bi-crystal systems to question a considerable variety of grain boundaries energy and excess volume. The hydrogen mobility and trapping process have been investigated based on the electrochemical hydrogen charging technique and on atomistic simulations using an Embedded Atom Method (EAM) potential. The confrontation of both technics allows to elucidate some relevant queries on the contribution of grain-boundary geometry to the mobility and trapping of hydrogen. The segregation process is discussed in relation to the systematic determination of short and longrange elastic distortions and the short-circuit of diffusion process is clarified with a confrontation of the different diffusion paths and the segregation energy of each grain boundaries considered. Both aspects offer new insight to disclose the impact of grain boundaries on some physical properties. 


\section{Results}

Some remarkable results from experimental works - Recently, an extensive collection of experimental data was gathered for the polycrystalline nickel to characterize diffusion and trapping processes which occur along grain boundaries ${ }^{9,27-29}$. This database is first revisited in the present work to introduce some open questions. The effective diffusion coefficient $\mathrm{D}_{\text {eff }}$ was assessed based on classical Fick's law to describe hydrogen flux across a polycrystalline membrane obtained during electrochemical permeation test. Using a large range of grain sizes it was possible to modulate the fraction of random grain boundary $\left(f_{\mathrm{R}}\right)$ and special grain boundary $\left(f_{\mathrm{CSL}}\right)^{27,28}$. A linear relationship is clearly established between $\operatorname{Ln}\left(\mathrm{D}_{\text {eff }}\right)$ and $f_{\mathrm{R}}$ the fraction of random GBs, (figure 1a) which illustrates the fact that random GBs can act as a short circuit of diffusion. In opposite, it was suggested that CSL GBs are specific locations for deep trapping based on the fact that the number of trapping sites $\mathrm{N}_{\mathrm{T}}$ increases as function with the fraction of CSL grain boundaries $\left(f_{\mathrm{CSL}}\right)$ (figure $11 \mathrm{~b}$ in Oudriss et al work ${ }^{28}$ ). Despite this demonstration of the global impact of both nature of GBs (Special and Random) on diffusion and trapping processes, some questions seem to be open. The separation of Special and Random class of GBs based only on the coincidence lower than $\Sigma 29$ seems unreasonable considering the energy of grain boundaries (see figure $2 \mathrm{a}$ as an example). Additionally, the hydrogen concentration $\mathrm{C}_{\mathrm{H}}$ increases with the fraction of random $f_{\mathrm{R}}$ which suggests that random GB is also a specific location of trapping. This effect was clearly identified as a consequence of a vacancy cluster formation (SAV) process. A linear relationship with vacancy concentration and hydrogen was identified $\left(\mathrm{C}_{\mathrm{vac}}=0.15 \times \mathrm{C}_{\mathrm{H}}{ }^{28}\right.$ which illustrates that the fact that the increase of hydrogen concentration is directly a consequence of vacancy formations without clearly establishing that the formation is directly promoted by random grain boundaries. The antagonist properties of trapping sites and short diffusion paths of random GBs illustrate some ambiguities of the interpretation of experimental data. More recently, we had the opportunity to use both TOF-SIMS and EBSD and combinate their analyses to retrieve the statistical information on the location of hydrogen near the GBs as a function of its character ${ }^{29}$. Initially, these data were only analyzed in term CSL $\Sigma 3^{\mathrm{n}}$ and random GBs, but in the present work, we show the opportunity to distinguish the coincident twin boundary CTB to other CSL boundaries (ATGB, asymmetric tilt grain boundary and SITB symmetric incoherent twin boundary). Figure $1 \mathrm{~b}$ illustrates the different profiles of hydrogen content observed around GBs after pre-charging and releasing steps which corresponds to a study state. A gap of hydrogen concentration between both adjacent grains is mostly related to random GBs. Consequently, it seems that when hydrogen diffusion occurs along GBs, hydrogen does not cross the GB easily 
but moves along the GB. In opposite, a constant profile where no significant modification of hydrogen content is mainly related to both grains around GB is observed for coincident twin boundary CTB where no defect and elastic distortion are necessary to accommodate the misorientation between adjacent grains.
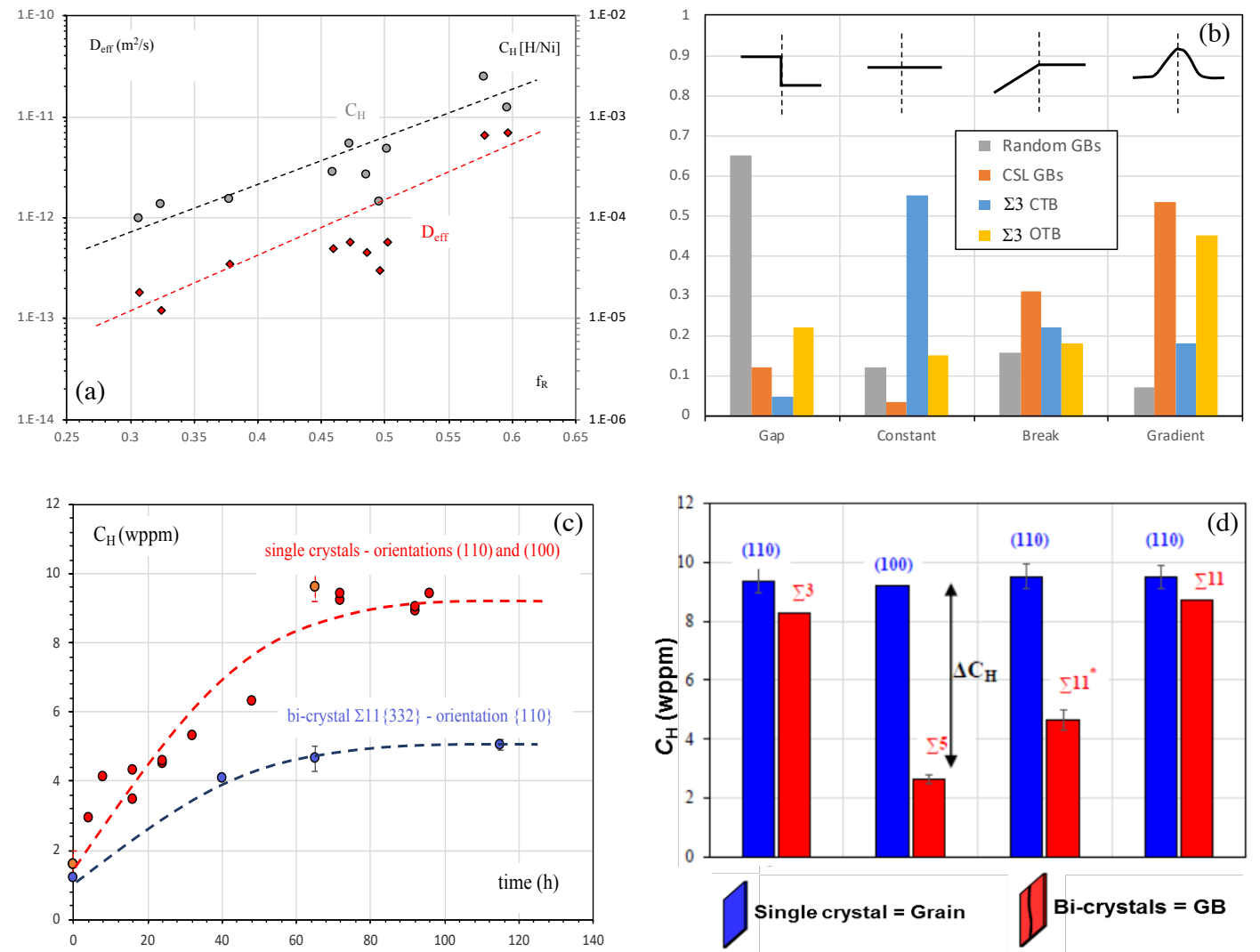

Figure 1 : Experimental data collected on the diffusion and solubility in nickel single and polycrystalline pure metal. (a) Effective diffusion coefficient Deff and hydrogen concentration $\mathrm{C}_{\mathrm{H}}$ as a function with the fraction of random grain-boundaries $f_{R}$ (data collected with Oudriss et al works ${ }^{28}$ electrochemical permeation for the same conditions and for different grain sizes between $10 \mathrm{~nm}$ to $200 \mu \mathrm{m}$ ). (b) Probability to obtain different gradient profiles of hydrogen concentration near GBs obtained by ToF-SIMS (data ${ }^{29}$ revisited, CTB Coherent Twin Boundary 53 , Other Twin Boundary 53 ). (c) Hydrogen concentration $\mathrm{C}_{\mathrm{H}}$ versus charging time for single crystal and $\Sigma 11-\{332\}$ bi-crystal with the hydrogen ingress orientation along $<110>$ (hydrogen charging at the cathodic current density of $5 \mathrm{~mA} / \mathrm{cm}^{2}$ in $0.1 \mathrm{M} \mathrm{NaOH}$ at $300 \mathrm{~K}$ ). (d) Hydrogen solubility $\mathrm{CH}_{\mathrm{H}}$ in single crystals and bi-crystals with different hydrogen ingress orientations: two orientations were studied: $\{110\}$ and $\{100\}$ (hydrogen charging at the cathodic current density of $5 \mathrm{~mA} / \mathrm{cm}^{2}$ in $0.1 \mathrm{M}$ $\mathrm{NaOH}$ at $300 \mathrm{~K}$ for 3 days, $\Sigma 3(\Sigma 3-\{111\} \mathrm{CTB}), \Sigma 5(\Sigma 5-\{310\}), \Sigma 11(\Sigma 11-\{311\})$ and $\Sigma 11^{*}(\Sigma 11-\{332\})$.

For the other CSL $\Sigma 3^{\mathrm{n}}$ boundaries OTB, a hydrogen gradient around GBs is observed where a large density of defects is necessary to accommodate the misorientation between two adjacent grains. Additionally, it was reported at a micrometer length scale of the gradient of hydrogen concentration significantly higher than GBs thickness (nanometer) ${ }^{29}$ which suggests the 
occurrence of the long-range internal stresses near OTB GBs. Considering these statistical results, we note that even though we associate a type of grain boundary type (random or special) with a hydrogen concentration profile, this result is by no means exclusive. This conclusion allows us to develop a work specifically on selected grain boundaries in a large domain of representative GBs. We have followed the hydrogen content as a function of charging time for a specific electrochemical charging condition which corresponds to a thermodynamic system defined by $\mathrm{P}_{\mathrm{H} 2}=800 \mathrm{~atm}$ and $\mathrm{T}=300 \mathrm{~K}$ on two systems single and bicrystal with the same ingress surface. The evolution of hydrogen concentration $\mathrm{C}_{\mathrm{H}}$ as a function of charging time is presented in figure $1 \mathrm{c}$ for the $\{110\}$ and $\{100\}$ single crystals and bicrystal $\Sigma 11\{332\}$ with a hydrogen ingress orientation of $\{110\}$. In each case, hydrogen concentration increases with time and reaches a saturation plateau corresponding to an apparent solubility. No significant difference is observed between both single crystals. In opposite the hydrogen content is largely lower in the considered bicrystal (5 wppm instead of 9 wppm for single crystals). We provide the same comparison for three other configurations in figure $1 \mathrm{~d}$ for the stationary state. A very low difference is observed between $\Sigma 3 \mathrm{CTB}$ and $\Sigma 11\{311\}$ in comparison with single crystal orientations. In opposite for $\Sigma 5\{310\}$ and $\Sigma 11\{332\}$ the hydrogen content obtained are lower than the one determined for equivalent single crystals. To resume, the hydrogen content is lower for bicrystals with an intensity defined by $\Delta \mathrm{C}_{\mathrm{H}}$ which depends on the grain boundary character and suggests that GBs act as a short-circuit of diffusion. The difference $\Delta \mathrm{C}_{\mathrm{H}}$ between bi-crystals and single crystal are questioned in term of the energy of grain boundary and the excess volume $V_{\text {ex. }}$. Both values are evaluated using atomistic calculation with EAM potential (see method) and confronted with data base of Olmsted et al. ${ }^{65}$ (figure 2a). Two domains corresponding to Special or CSL GBs and Random GBs are represented in this figure. Additionally, our studied GBs are defined with red dots. In a specific range of energy and excess volume (intersection between two domains), it is impossible to distinguish both kinds of GBs (CSL or Random). Moreover, for the GBs selected in the present work a linear relation can be found between $\mathrm{E}_{\mathrm{GB}}$ and $\mathrm{V}_{\mathrm{ex}}: E_{G B} \approx 3.32 \times V_{\text {ex }}$ despite the fact that we observed a large scatter in the figure $2 \mathrm{a}$. The possible correlation between the excess volume $\mathrm{V}_{\mathrm{ex}}$ and the difference of solubility $\Delta \mathrm{C}_{\mathrm{H}}$ is evaluated in figure $2 \mathrm{~b}$ and allows suggesting that a higher disorder in grain-boundary (higher excess volume) promotes the hydrogen diffusion along grain boundaries. Based on these experimental data, the understanding of the competition between hydrogen segregation and diffusion along the grain-boundaries stays a challenge. 

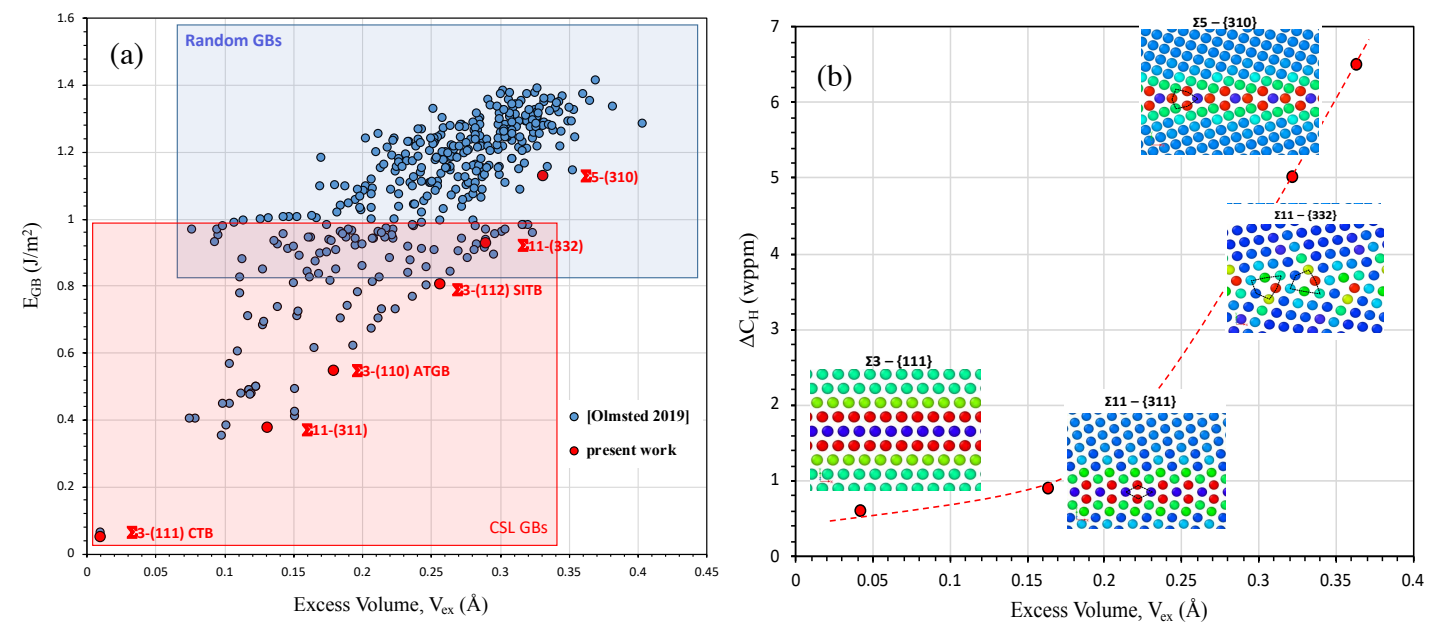

Figure 2 : (a) Grain-boundaries energy $\mathrm{E}_{\mathrm{GB}}$ versus the excess volume $\mathrm{V}_{\mathrm{ex}}$ (data from atomistic calculations courtesy to Olmsted et al. ${ }^{65}$ ). The grains boundaries studies in present work are represented in the picture with red dots. Our calculation has been performed with the same atomic potential. (b) the difference of hydrogen solubility $\Delta \mathrm{CH}_{\mathrm{H}}$ between single crystal and bi-crystal with the same hydrogen ingress orientation as a function with the grain boundary excess volume $\mathrm{V}_{\mathrm{ex}}\left(\Delta \mathrm{CH}\right.$ are collected by experimental works, $\mathrm{V}_{\text {ex }}$ is determined numerically using atomistic code).

Segregation energy versus grain-boundary character-The segregation energy for the different sites in and near the GBs has been determined for the four GBs studied. According to previous works (see as examples ${ }^{47,55,66,67}$ ), the segregation energy of hydrogen in a specific location in and near GBs is relatively reported to the octahedral site since it is the most stable one in fcc nickel phase (the insertion energy for octahedral site is $E_{o c t}^{i n s}=0.1775 \mathrm{eV}$ while for tetrahedral site it is equal to $0.586 \mathrm{eV}$ ). The energy can be represented as a function of the distance from the GB plane (figure 3a). Since a large variety of segregation energy can be obtained for some GBs and specifically for large excess volume (see $\Sigma 11-\{332\}$ as an example) we characterise this distribution by the maximum of this energy $\mathrm{E}_{\mathrm{seg}(\max )}$ and the thickness, $\mathrm{e}_{\mathrm{GB}}$ of GBs defined from an energy point of view. $\mathrm{e}_{\mathrm{GB}}$ characterises a length scale of the impact of $\mathrm{GB}$ on the segregation of hydrogen. $\mathrm{E}_{\mathrm{seg}(\max )}$ is obtained in the GBs core and depends on the GB character. Both parameters $\left(\mathrm{E}_{\mathrm{seg}(\max )}\right.$ and $\mathrm{e}_{\mathrm{GB}}$ ) are an increasing function of the grain boundary energy, $\mathrm{E}_{\mathrm{GB}}$ (figure $3 b)$. According to the linear relation between grain boundary energy and the excess volume $V_{e x}$ (figure 2a), the last results can be interpreted as a consequence of the disorder impact on the intensity of segregation process and the domain of the occurrence. To question the possible implication of this disorder on the hydrogen segregation site near grain boundaries, we characterise precisely each location of hydrogen segregation firstly by the hydrogen atomic volume, $\mathrm{V}_{\mathrm{H}}$. This volume defined by neighbouring nickel atoms is calculated using the Voronoi method. The hydrogen atomic Voronoi volume at the bulk octahedral site $V_{\text {Oct }}$ is $5.773 \AA^{3}$ and presents a cubic morphology. All segregation sites contiguous to the GB region have a higher atomic volume and a more stable segregation state (figure 3d). Concerning the lower energy GBs, 
the segregation energy $E_{\text {seg }}$ is a quite linear function of the hydrogen atomic volume, $V_{H}$ (domain I). However, this linear relationship is not available for high energy GBs with an atomic volume above $6.6 \AA^{3}$ (domain II) in accordance with our previous work related to $\Sigma 3^{\mathrm{n}}$ special $\mathrm{GBs}^{47}$.


Figure 3 : (a) Hydrogen segregation energy, Eseg as a function of the hydrogen location in the grain boundary core region. (b) Grain boundary thickness, e and maximum hydrogen segregation energy, $E_{s e g}(\max ) v s$ the

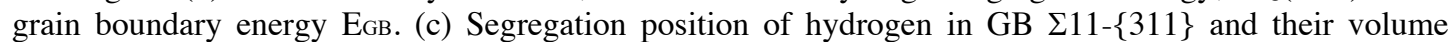
geometry defined by Voronoi's method. (d) Segregation energy as a function of the hydrogen atomic volume $\mathrm{V}_{\mathrm{H}}$ for the different locations in the seven grain boundaries studied.

In domain II, a quasi-plateau is reached in terms of energy $(-0.22 \mathrm{eV})$ with a large scatter. Consequently, the hydrogen atomic volume seems to be insufficient to question the elastic energy contribution to the segregation energy which allows in the following to consider the morphology of the different sites. The segregation sites in the GB region have complicated local geometry structures, thus, we will describe the geometry of all the potential segregation sites in GBs core in detail. An illustration of this approach is shown in figure $3 \mathrm{c}$ where the segregation positions and their volume geometry are presented for the $\Sigma 11-\{332\}$ GB. The position numbers are ranked from the most to less stable segregation energy. According to the Voronoi tessellation, the 
hydrogen volume at the octahedral site in nickel bulk is a cubic form with 14 neighbouring atoms. The closer the hydrogen atoms get to the GB core region, the greater the geometry deformation occurs. Consequently, the segregation energy and the hydrogen volume size are extremely dependent on the local environment. However, a direct relationship cannot be found among these factors. The morphology of the different sites highlights the fact that the deformation of the site is not isotropic for most cases. We will discuss this crucial aspect in the next section. After the consideration of the local volume deformation, the hydrogen atom insertion at different sites can be discussed in terms of local energy of hydrogen $E_{H}$ (the energy of atom $i$ in the EAM method with $\mathrm{i}=\mathrm{H}$ ). This energy is the sum of the kinetic and the potential energy, which differs from the segregation energy $E_{\text {seg }}$ only if we have a long-range effect associated with the insertion of hydrogen. Figure $4 \mathrm{a}$ shows the relationships between the segregation energy, $\mathrm{E}_{\text {seg }}$ and the hydrogen atom energy, $\mathrm{E}_{\mathrm{H}}$. Two domains are clearly observed in relation with the ones defined in figure $3 \mathrm{~d}$. For low hydrogen atom energy, a linear relation with segregation energy is clearly established $\left(\mathrm{E}_{\mathrm{seg}}=0.54 \times \mathrm{E}_{\mathrm{H}}+0.0864\right)$ which corresponds to a range of low hydrogen atomic volume range $\mathrm{V}_{\mathrm{H}}<6.6 \AA^{3}$ (domain I). The slope 0.54 of $\mathrm{E}_{\text {seg }}$ versus $\mathrm{E}_{\mathrm{H}}$ coincides to the ratio between the unstrained atomic volume $\Omega_{\mathrm{Ni}}\left(10.904 \AA^{3}\right)$ and one of the unstrained octahedral interstitial sites $\mathrm{V}_{\text {Oct }}\left(5.773 \AA^{3}\right)$. Consequently, the hydrostatic deformation induced by the incorporation of hydrogen is a second order term for the relationship between $\mathrm{E}_{\mathrm{seg}}$ and $\mathrm{E}_{\mathrm{H}}$. For a segregation energy equal to zero $\left(\mathrm{E}_{\mathrm{seg}}=0 \mathrm{eV}\right)$, the hydrogen atom energy, $\mathrm{E}_{\mathrm{H}}$ is equal to $0.16 \mathrm{eV}$ which is quite similar to the value of the insertion energy for octahedral site $E_{o c t}^{i n s}(0.1775 \mathrm{eV})$. For a higher value of $E_{H}$ above $-0.5 \mathrm{eV}$ (domain II) a large scatter demonstrates that probably long-range displacements of the nickel atoms occur in addition to the short-range displacement of the neighbouring atoms. Concerning figure $3 \mathrm{~d}$, the picture highlights the fact that hydrogen atom is more stable at a deep segregation site with high atomic volume. Actually, we have noticed that a part of the data has a linear evolution $\left(E_{\text {seg }} \sim A \times\left[V_{H}-V_{\text {oct }}\right]\right.$ with a slope A equal to 0.43$)$. These sites have a similar cubic form with a relatively low hydrogen atomic volume. This linearity disappears when the distortion becomes significant $\left(\mathrm{V}_{\mathrm{H}}>6.6 \AA^{3}\right)$ and probably with a long-range impact of this distortion (see the next section). Moreover, instead of domain II, we observed some specific situations as the fact that some sites where hydrogen atomic volume $\mathrm{V}_{\mathrm{H}}$ is lower than the one of the octahedral interstitial sites $V_{\text {Oct }}\left(5.773 \AA^{3}\right)$. These situations have been recently related near a free surface of nickel single crystal ${ }^{102}$.

\section{Segregation energy versus elastic energy}

As previously highlighted in different configurations, the insertion of a solute in a specific location induces a geometric transformation which cannot be only described as a volume change (see as examples ${ }^{16,47,68-73}$ ). The modification of the geometric configuration of the volume defined 
by Voronoi's method results in an equilibrium with the different interactions between the neighboring atoms of the solute. The contribution of the elastic strain energy of this process can be evaluated by considering the respective effect of hydrostatic strain (change of the volume) and shear strain (change of the morphology, deviatoric part of strain). In order to investigate the contribution of the elastic strain energy to the segregation process the elastic dipole tensor $\mathrm{P}_{\mathrm{ij}}$ is used to describe the equilibrium in a continuum solid of a point-force distribution ${ }^{47,73-75}$. The elastic dipole tensor characterizes the changes for both the volume and the shape of the interstitial site during the relaxation procedure after the incorporation of the solute in the lattice. From lattice point defect distortion theory, the dipole tensor is given by:

$P_{i j}=\sum_{m} f_{j}^{m / s o l} r_{i}^{m}$

where $f_{j}^{m / s o l}$ is the force exerted from the solute surrounding atoms $\mathrm{m}$ and $r_{i}^{m}$ is the atomic displacement of atoms. In a first approach, the lattice distortion around the solute is supposed to occur mainly on the insertion site closest to the neighboring atoms. Thus, in isotropic medium, the stress $\sigma_{i j}$ and the elastic strain energy $\mathrm{E}_{\mathrm{el}}$ by unit volume induced from the insertion of the hydrogen interstitial are related to the dipole tensor ${ }^{47}$ :

$\sigma_{i j}=\frac{P_{i j}}{\mathrm{~V}_{H}}=\sigma_{m} \delta_{i j}+\sigma_{i j}^{d}$ and $E_{e l}=E_{e l}^{m}+E_{e l}^{d}=\frac{1}{2 B}\left(\sigma_{m}\right)^{2}+\frac{1}{6 G}\left(\sigma_{d}\right)^{2}$

Where $\sigma_{m}$ and $\sigma_{d}$ are respectively the hydrostatic stress $\left(\frac{1}{3}\left[\sigma_{11}+\sigma_{22}+\sigma_{33}\right]\right)$ and the deviatoric stress defined as Von Mises equivalent stress $\left(2 \sigma_{d}^{2}=\left(\sigma_{11}-\sigma_{22}\right)^{2}+\left(\sigma_{11}-\sigma_{33}\right)^{2}+\left(\sigma_{33}-\right.\right.$ $\left.\left.\sigma_{22}\right)^{2}+6\left(\sigma_{12}^{2}+\sigma_{23}^{2}+\sigma_{13}^{2}\right)\right)$. B and $\mathrm{G}$ are the bulk and shear modulus respectively. $E_{e l}^{m}$ and $E_{e l}^{d}$ represent the dilatational and distortion energies. The evolution of hydrostatic and deviatoric part of stress field as a function of hydrogen atomic volume is illustrated in figure 4b. Two behaviors can be distinguished: in domain I, for a lower value of $\mathrm{V}_{\mathrm{H}}$ below $6.6 \AA^{3}$, the hydrostatic component is predominant, whereas in domain II both stresses have the same order of intensity. The direct consequence of this result is observed in the correlation between the local energy of hydrogen $E_{H}$ and the elastic strain energy $E_{\text {el }}$ (figure $4 c$ ). This one illustrates a change of behavior between both domains defined previously. In domain I, a linear regime between both energies is observed with a transition around a strain energy density of about $2 \mathrm{GPa}$. Below this value a nonlinear evolution is established and referred to as domain II. As it is illustrated by the figure $4 \mathrm{~d}$, the elastic strain energy $E_{e l}$ is dominated by the hydrostatic stress for the first linear regime $\left(E_{e l} \approx\right.$ $\frac{1}{2 B}\left(\sigma_{m}\right)^{2}$ in domain $\mathrm{I}, \mathrm{E}_{\mathrm{el}}>2 \mathrm{GPa}, \mathrm{E}_{\mathrm{H}}>-0.5 \mathrm{eV}$ and $\mathrm{V}_{\mathrm{H}}<6.6 \AA^{3}$ ) while the other regime (domain 
II, $\mathrm{E}_{\mathrm{el}}<2 \mathrm{GPa}, \mathrm{E}_{\mathrm{H}}<-0.5 \mathrm{eV}$ and $\mathrm{V}_{\mathrm{H}}>6.6 \AA^{3}$ ) is due to the contribution of the deviatoric and hydrostatic stresses with a similar contribution for the large volumes.
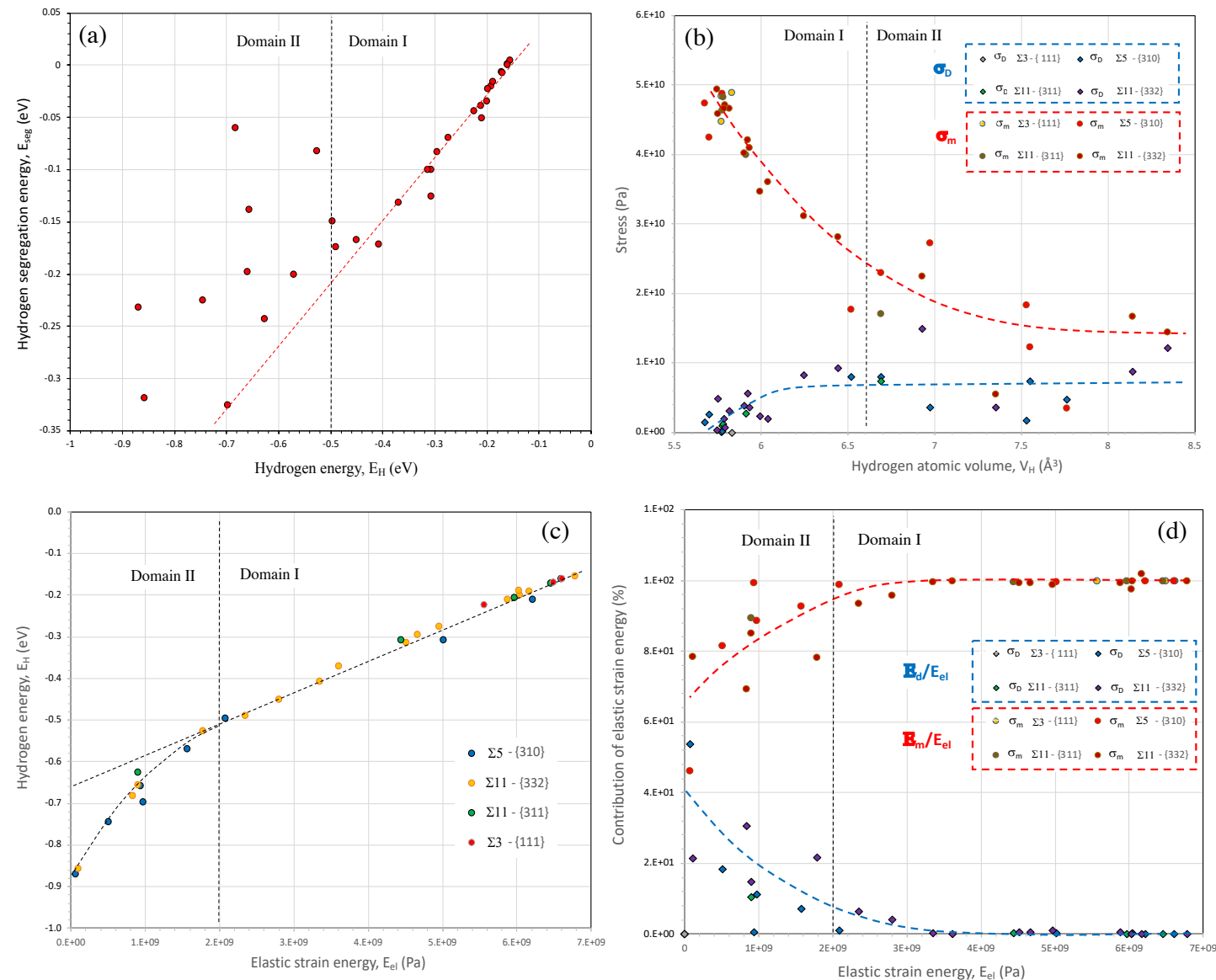

Figure 4 : (a) Hydrogen segregation energy Eseg versus hydrogen energy Ен. (b) Hydrostatic $\sigma_{\mathrm{m}}$ and deviatoric stress $\sigma_{D}$ as a function of hydrogen atomic volume $\mathrm{V}_{\mathrm{H}}$. (c) Hydrogen energy EH $v_{s}$ the elastic strain energy Eel (per unit of volume) (d) Contributions to the hydrostatic, EH and deviatoric, ED parts of energy to the elastic strain energy Eel.

Assuming that local energy of hydrogen is dominated by the elastic strain energy induced by the insertion of the solute, the slope of linear behavior observed in domain I between hydrogen energy, $\mathrm{E}_{\mathrm{H}}$ and the elastic strain energy per unit volume, $\mathrm{E}_{\mathrm{el}}$ (figure $4 \mathrm{c}$ ) is directly associated with an apparent hydrogen volume $V_{H}^{a p}$ equal to $12 \AA^{3}$. In regime $\mathrm{I}$, the hydrogen volume $\mathrm{V}_{\mathrm{H}}$ stays equal to the hydrogen volume of the bulk octahedral interstitial site $\left(5.773 \AA^{3}\right)$ with only a difference lower than $10 \%$. The discrepancy observed between $V_{H}^{a p}$ and $\mathrm{V}_{\mathrm{H}}$ is directly associated with the fact that firstly (eq. 2) we do not consider the impact of hydrogen on the elastic properties. The degradation of elastic properties correlated to the incorporation of hydrogen is known well documented in the literature $\mathrm{e}^{17,73,76-81}$. 
Based on the linear-elastic theory of the equilibrium between interstitial solute and surrounding metal lattice, the apparent bulk modulus $\mathrm{B}^{*}$ can be determined considering the Eshelby's inclusion model in case of isotropic elastic properties of a solid ${ }^{76,77}: B^{*}=B\left[\frac{2(1-2 v)}{(1+v)}\right]$. Consequently, considering elastic strain energy $E_{\mathrm{el}}$ dominated by the hydrostatic stress with the bulk modulus correction we find $V_{H}^{a p}=6.5 \AA^{3}$ which is in accordance with $\mathrm{V}_{\mathrm{H}}$ obtained in regime I (between 5.7 to $6.4 \AA^{3}$ ). In agreement with the figure $4 \mathrm{c}$, this result indicates that the local energy of hydrogen $\mathrm{E}_{\mathrm{H}}$ verifies that:

$E_{H}=E_{e l}^{m}+E^{\text {chem }}$

with a dilatational energy equal to : $E_{e l}^{m}=\frac{(1+v)}{4 B(1-2 v)}\left(\sigma_{m}\right)^{2} V_{H}$.

The second term of the equation eq. $3, E^{\text {chem }}$ is the electronic contribution or "chemical" term which corresponds to the embedding energy due to the distortion of chemical bonds near the solute ( $E^{\text {chem }}=-0.653 \mathrm{eV}$ ). Equation (3) can be extended in regime II, considering the increase of the hydrogen atomic volume and the implication of distortion part of the elastic energy $E_{e l}^{d}$. For the last component, we also consider the degradation of elastic properties with hydrogen $\left(G^{*}=3 B^{*}\left[\frac{(1-2 v)}{2(1+v)}\right]\right)$. In domain II, $\mathrm{V}_{\mathrm{H}}$ increases with the decrease of the elastic energy to reach a value near $8.2 \AA^{3}$. This value is very close to the atomic volume of hydrogen localized in a vacancy $V_{v a c}^{H}\left(\sim 8.66 \AA^{3}\right)^{47}$ where the contribution of elastic energy is negligible instead of an electronic contribution in accordance with the present results ${ }^{82}$. The equation (3) can be rewritten considering the previous comment as:

$E_{H}=E_{e l}^{m}+E_{e l}^{d}+E_{c h e m}$

with $E_{e l}^{d}=\frac{1}{6 G^{*}}\left(\sigma_{d}\right)^{2} V_{H}$.

This equation predicts that for total elastic energy $E_{e l}=E_{e l}^{m}+E_{e l}^{d}=0 \mathrm{eV}$ that $E_{H}=E_{\text {chem }}=-$ $0.653 \mathrm{eV}$ which is higher than the one related in figure $4 \mathrm{c}$ in domain II $(-0.85 \mathrm{eV})$. This difference suggests that the contribution of the electronic component of energy increases in domain II when the hydrogen volume increases to a value near $V_{v a c}^{H}$. In the present work, the elastic contribution of the energy of hydrogen $\mathrm{E}_{\mathrm{H}}$ is between $0.286 \mathrm{eV}$ and $0.082 \mathrm{eV}$ in regime I and lower than 0.082 $\mathrm{eV}$ in regime II. The main effect is consequently observed in regime I where the hydrostatic strain contribution is predominant and has the same order of magnitude as the chemical contribution.

Both figures $3 \mathrm{~d}\left(E_{\text {seg }} v s V_{H}\right)$ and $4 \mathrm{a}\left(E_{s e g} v s E_{H}\right)$ clearly demonstrate that a significant number of insertion sites do not verify the relationships previously defined. These situations correspond to sites of the grain-boundaries with higher energy and excess volume $(\Sigma 11-\{332\}$ and $\Sigma 5-\{310\})$. 
The fact that we cannot find a relation with $E_{s e g}$ and $E_{H}$ or $V_{H}$, suggesting that a significant part of the segregation energy depends on the long-range elastic distortion. To support this idea, two situations are illustrated in figure 5. The first one corresponds to the location (a) where a longrange displacement is observed along the axis $<110>$ and a short-range displacement in any other directions (Figure 5a). The profile of the radial deformation is shown in figure $5 \mathrm{~b}$ with a length scale of around $\sim 8 \AA$ and an amplitude of $16 \%$. The first neighbor's energy (1 to 4 ) corresponds to a long-range elastic interaction $\mathrm{E}_{\mathrm{el}}^{\mathrm{LR}} \sim 4.310^{9} \mathrm{~Pa}$ (or $0.16 \mathrm{eV}$ ) which needs to be integrated in the total strain energy density. With this contribution the location (a) follows the linear curve $\mathrm{E}_{\mathrm{seg}} v s \mathrm{E}_{\mathrm{el}}$ defined in domain I with $E_{e l}=E_{e l}^{d}+E_{e l}^{m}+\mathrm{E}_{\mathrm{el}}^{\mathrm{LR}}$. The second situation illustrated in figure $5 \mathrm{~d}$ is the location (b) in domain II which verifies the linear curve $\mathrm{E}_{\mathrm{seg}} v s \mathrm{E}_{\mathrm{el}}$ without any addition of long-range elastic interactions term because quasi-isotropic short-range displacements are only observed. Consequently, the large scattering observed in figure 4a results from the longrange elastic energy between $0.08 \mathrm{eV}$ and $0.44 \mathrm{eV}$.
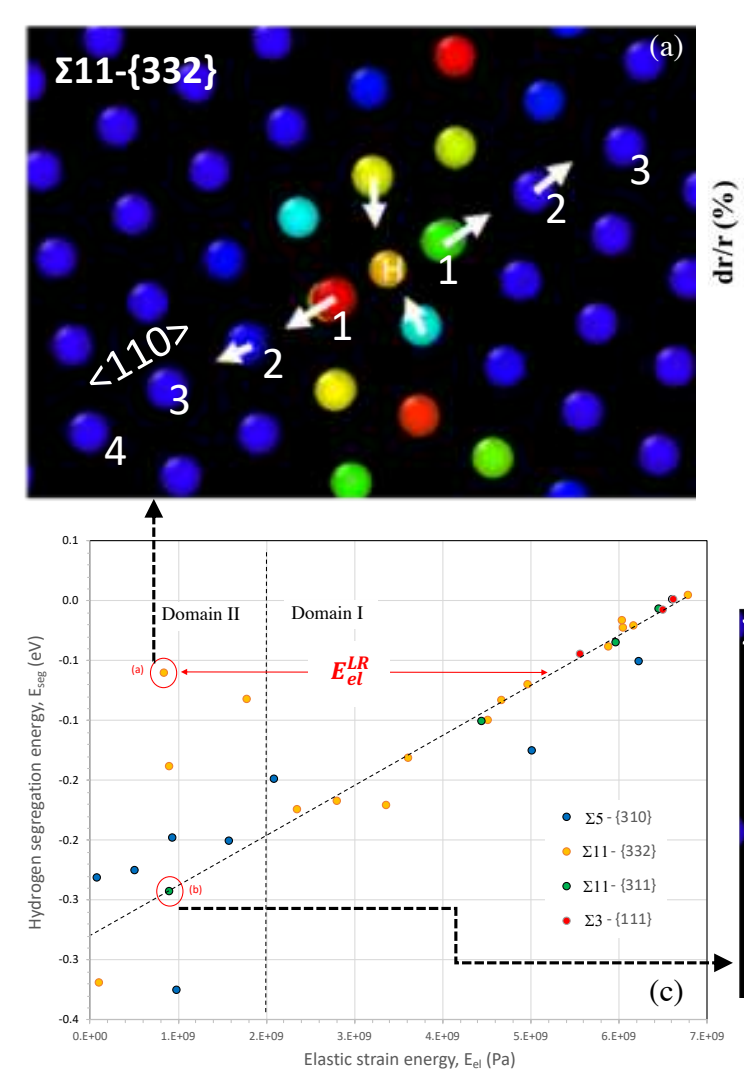

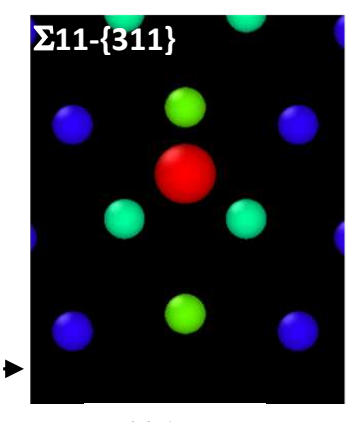

Initial state

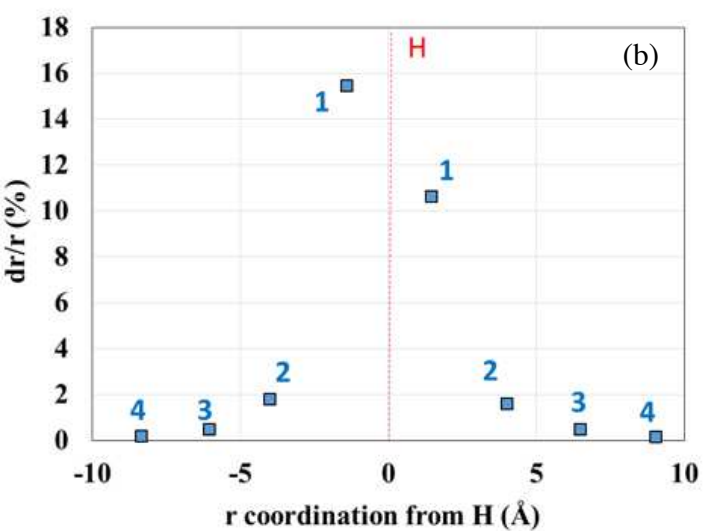

r coordination from $\mathbf{H}(\AA)$



Final state

(d)

Figure 5 : The loss of the linear relation between the segregation energy, Eseg and the elastic strain energy $\mathrm{Eel}(\mathrm{c})$ is directly a consequence of the anisotropy of the displacement field and "long range" elastic distortion. (a) An illustration of the anisotropy of the displacement field along $<110>$ near a $\Sigma 11-\{332\}$. (b) the relative radial displacement $\mathrm{dr} / \mathrm{r}$ along $<110>$ as a function of the $\mathrm{r}$ coordination from $\mathrm{H}$ (corresponding to the $\Sigma 11-\{332\}$ illustrated in (a)). (d) Moderate quasi-isotropic short range displacement for a position of hydrogen which respects the linear relation of Eseg versus Eel (see picture (c), GB $\Sigma 11-\{311\}$ ). 


\section{Diffusion paths}

The short circuit of diffusion along GBs can be discussed as a function with a reference state defined as a diffusion path of the perfect crystal. The nudged elastic band method (NEB) method has been used to calculate the Minimum Energy Paths (MEP) and their associated energy barriers (Table 1). Several stable segregation sites in GBs were looked upon for three principal path directions: (i) the hydrogen atom moves from the bulk site to the GB site (figure 6a), (ii) the hydrogen atom moves between two GB sites along the GB plane (figure 6b), (iii) the hydrogen atom moves between two GB sites along the tilt axis (figure 6c). The first investigation of the migration energy $E_{m}$ has been carried out in the perfect nickel crystal. For the easier path, the hydrogen atom moves between octahedral sites $(\mathrm{O})$ through a metastable tetrahedral site $(\mathrm{T})$ in nickel bulk and the energy barriers, $E_{m}^{0-T}$ is equal to $0.47 \mathrm{eV}$ in accordance with the DFT works: $\sim 0.46 \mathrm{eV}^{83}$ and $\sim 0.41 \mathrm{eV}^{84}$. This migration energy is defined as the reference state, the hydrogen atom is able to move faster if the energy barrier is lower than this reference energy. The movement of a hydrogen atom in the GB core and cross the GB is illustrated in figure 6 where it is given the MEP and the energy barriers of NEB calculations. The energy barrier is calculated with the initial position energy as the reference point. We have considered several positions such as the most stable segregation sites (type A) and the highest volume sites (type B). A and B positions are the same only for the GBs $\Sigma 11-\{311\}$ and $\Sigma 3-\{111\}$. In each situation, we have considered the forward $\left(E_{m}^{F}\right)$ and backward $\left(E_{m}^{B}\right)$ paths. All the energies are reported in table 1.

Table 1 : Energy barriers for the most stable segregation position A and the highest volume position $B$ along the different directions $\mathrm{X} ; \mathrm{Y}$ and $\mathrm{Z}$. The forward $\left(E_{m}^{F}\right)$ and backward $\left(E_{m}^{B}\right)$ paths are differentiated.

\begin{tabular}{|c|c|c|c|c|}
\hline Grain-boundaries & Segregation site & X, along GB & Y, across GB & Z, along GB \\
\hline$\Sigma 3-\{111\}$ CTB & $\mathrm{A} / \mathrm{B}$ & $\mathrm{E}_{\mathrm{m}}^{\mathrm{F}}=\mathrm{E}_{\mathrm{m}}^{\mathrm{B}}=0.52 \mathrm{eV}$ & $\mathrm{E}_{\mathrm{m}}^{\mathrm{F}}=\mathrm{E}_{\mathrm{m}}^{\mathrm{B}}=0.46 \mathrm{eV}$ & - \\
\hline 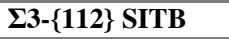 & $\mathrm{A} / \mathrm{B}$ & $\mathrm{E}_{\mathrm{m}}^{\mathrm{F}}=\mathrm{E}_{\mathrm{m}}^{\mathrm{B}}=0.6 \mathrm{eV}$ & $\mathrm{E}_{\mathrm{m}}^{\mathrm{F}}=0.42 \mathrm{eV} / \mathrm{E}_{\mathrm{m}}^{\mathrm{B}}=0.65 \mathrm{eV}$ & - \\
\hline$\Sigma 11-\{311\}$ & $\mathrm{A} / \mathrm{B}$ & $\mathrm{E}_{\mathrm{m}}^{\mathrm{F}}=\mathrm{E}_{\mathrm{m}}^{\mathrm{B}}=0.51 \mathrm{eV}$ & $\mathrm{E}_{\mathrm{m}}^{\mathrm{F}}=0.47 \mathrm{eV} / \mathrm{E}_{\mathrm{m}}^{\mathrm{B}}=0.72 \mathrm{eV}$ & $\mathrm{E}_{\mathrm{m}}^{\mathrm{F}}=\mathrm{E}_{\mathrm{m}}^{\mathrm{B}}=0.6 \mathrm{eV}$ \\
\hline \multirow[t]{2}{*}{$\mathbf{\Sigma 1 1}-\{332\}$} & $\mathrm{A}$ & $\mathrm{E}_{\mathrm{m}}^{\mathrm{F}}=\mathrm{E}_{\mathrm{m}}^{\mathrm{B}}=0.61 \mathrm{eV}$ & $\mathrm{E}_{\mathrm{m}}^{\mathrm{F}}=0.48 \mathrm{eV} / \mathrm{E}_{\mathrm{m}}^{\mathrm{B}}=0.81 \mathrm{eV}$ & $\mathrm{E}_{\mathrm{m}}^{\mathrm{F}}=\mathrm{E}_{\mathrm{m}}^{\mathrm{B}}=0.23 \mathrm{eV}$ \\
\hline & B & $\mathrm{E}_{\mathrm{m}}^{\mathrm{F}}=\mathrm{E}_{\mathrm{m}}^{\mathrm{B}}=0.3 \mathrm{eV}$ & $\mathrm{E}_{\mathrm{m}}^{\mathrm{F}}=0.46 \mathrm{eV} / \mathrm{E}_{\mathrm{m}}^{\mathrm{B}}=0.53 \mathrm{eV}$ & $\mathrm{E}_{\mathrm{m}}^{\mathrm{F}}=\mathrm{E}_{\mathrm{m}}^{\mathrm{B}}=0.22 \mathrm{eV}$ \\
\hline \multirow[t]{2}{*}{$\Sigma 5-\{310\}$} & A & $\mathrm{E}_{\mathrm{m}}^{\mathrm{F}}=\mathrm{E}_{\mathrm{m}}^{\mathrm{B}}=0.51 \mathrm{eV}$ & $\mathrm{E}_{\mathrm{m}}^{\mathrm{F}}=0.46 \mathrm{eV} / \mathrm{E}_{\mathrm{m}}^{\mathrm{B}}=0.79 \mathrm{eV}$ & $\mathrm{E}_{\mathrm{m}}^{\mathrm{F}}=\mathrm{E}_{\mathrm{m}}^{\mathrm{B}}=0.5 \mathrm{eV}$ \\
\hline & B & $\mathrm{E}_{\mathrm{m}}^{\mathrm{F}}=\mathrm{E}_{\mathrm{m}}^{\mathrm{B}}=0.3 \mathrm{eV}$ & $\mathrm{E}_{\mathrm{m}}^{\mathrm{F}}=0.46 \mathrm{eV} / \mathrm{E}_{\mathrm{m}}^{\mathrm{B}}=0.7 \mathrm{eV}$ & $\mathrm{E}_{\mathrm{m}}^{\mathrm{F}}=\mathrm{E}_{\mathrm{m}}^{\mathrm{B}}=0.45 \mathrm{eV}$ \\
\hline
\end{tabular}

For the GB $\Sigma 3-\{111\}$ according to the migration energy, a hydrogen atom can easily cross the $\mathrm{GB}\left(E_{m}^{G B, Y}=0.46 \mathrm{eV} \sim E_{m}^{0-T}=0.47 \mathrm{eV}\right)$ but it is slightly slowed down along the GB plane $\left(E_{m}^{G B, X}=0.52 \mathrm{eV}>E_{m}^{0-T}=0.47 \mathrm{eV}\right)$.

For the low energy/excess volume GB $\Sigma 11-\{311\}$, the hydrogen atom can move easily from the bulk to the $\mathrm{GB}$ core $\left(\mathrm{E}_{\mathrm{m}}^{\mathrm{GB}, \mathrm{Y}}=\mathrm{E}_{\mathrm{m}}^{\mathrm{F}}=0.47 \mathrm{eV}=\mathrm{E}_{\mathrm{m}}^{0-\mathrm{T}}\right)$, but the backward path is more difficult $\left(\mathrm{E}_{\mathrm{m}}^{\mathrm{GB}, \mathrm{Y}}=\mathrm{E}_{\mathrm{m}}^{\mathrm{B}}=0.72 \mathrm{eV}>\mathrm{E}_{\mathrm{m}}^{0-\mathrm{T}}\right)$ as are the other directions $\left(\mathrm{E}_{\mathrm{m}}^{\mathrm{GB}, \mathrm{X}}=0.51 \mathrm{eV}\right.$ and $\mathrm{E}_{\mathrm{m}}^{\mathrm{GB}, \mathrm{Z}}=$ $0.6 \mathrm{eV}$ ) where the diffusion gets slower in the GB core. This GB has a few numbers of sites for the hydrogen segregation (four sites) but its segregation sites are probably trapping sites. 
The GB $\Sigma 11-\{332\}$ is a high energy/excess volume GB (figure 6). It has several hydrogen segregations sites in the GB core (14 sites). For the segregation site A, the energy barrier along the $\mathrm{X}$ direction is higher than the reference energy in nickel bulk $\left(E_{m}^{G B, X}=0.61 \mathrm{eV}>E_{m}^{0-T}=\right.$ $0.47 \mathrm{eV}$ ), in opposite for the segregation site $\mathrm{B}$ where we observed a fast diffusion path with lower migration energy $\left(E_{m}^{G B, X}=0.3 \mathrm{eV}<E_{m}^{0-T}=0.47 \mathrm{eV}\right)$. A quite similar value for the migration energy along $\mathrm{Z}$ direction is obtained for the segregation sites $\mathrm{A}$ and $\mathrm{B}\left(\mathrm{E}_{\mathrm{m}}^{\mathrm{GB}, \mathrm{Z}}\right.$ equal 0.23 $\mathrm{eV}$ for $\mathrm{A}$ site and $0.22 \mathrm{eV}$ for $\mathrm{B}$ site) which allows to define this direction as a fast diffusion path. For the cross direction $\mathrm{Y}$, the hydrogen atom can move easily from the bulk to the GB core ( $\mathrm{E}_{\mathrm{m}}^{\mathrm{GB}, \mathrm{Y}}=\mathrm{E}_{\mathrm{m}}^{\mathrm{F}}$ is equal to $0.48 \mathrm{eV}$ for $\mathrm{A}$ site and $0.46 \mathrm{eV}$ for $\mathrm{B}$ site ), but the backward path is more difficult $\left(\mathrm{E}_{\mathrm{m}}^{\mathrm{GB}, \mathrm{Y}}=\mathrm{E}_{\mathrm{m}}^{\mathrm{B}}\right.$ is equal to $0.81 \mathrm{eV}$ and $0.53 \mathrm{eV}$ respectively for $\mathrm{A}$ and $\mathrm{B}$ sites).

The GB $\Sigma 5-\{310\}$ is the highest energy/excess volume GB in our samples, it has less segregation sites than the GB $\Sigma 11-\{332\}$ in the GB core (8 sites). Along the X_direction, it exists a diffusion path for which hydrogen atom moves faster at the highest volume sites $B\left(E_{m}^{G B, X}=0.3 e V<\right.$ $\mathrm{E}_{\mathrm{m}}^{0-\mathrm{T}}=0.47 \mathrm{eV}$ ). However, the diffusion at the most stable segregation sites A slows down $\left(\mathrm{E}_{\mathrm{m}}^{\mathrm{GB}, \mathrm{X}}=0.51 \mathrm{eV}>\mathrm{E}_{\mathrm{m}}^{0-\mathrm{T}}=0.47 \mathrm{eV}\right)$ and can be considered as a trapping site. Along the $\mathrm{Z}$ direction, the energy barrier higher than the reference energy in nickel bulk and cannot be considered as a new path of diffusion (table 1). For the cross direction $\underline{Y}$, the hydrogen atom can move easily from the bulk to the $\mathrm{GB}$ core $\left(\mathrm{E}_{\mathrm{m}}^{\mathrm{GB}, \mathrm{Y}}=\mathrm{E}_{\mathrm{m}}^{\mathrm{F}}=0.46 \mathrm{eV}\right.$ for for $\mathrm{A}$ and $\mathrm{B}$ sites), but the backward path is more difficult $\left(\mathrm{E}_{\mathrm{m}}^{\mathrm{GB}, \mathrm{Y}}=\mathrm{E}_{\mathrm{m}}^{\mathrm{B}}\right.$ is equal to $0.79 \mathrm{eV}$ and $0.7 \mathrm{eV}$ respectively for $\mathrm{A}$ and B sites) which promotes the stabilization of hydrogen on GB. 
(a)

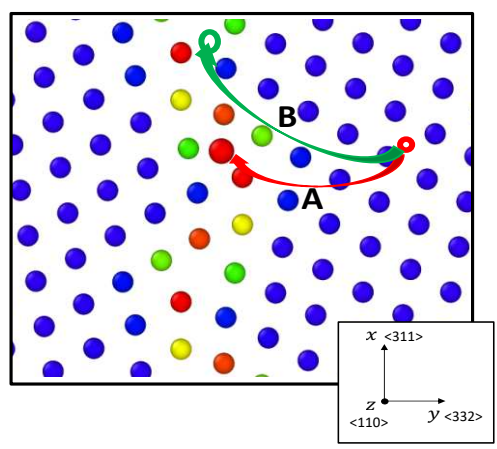

(b)

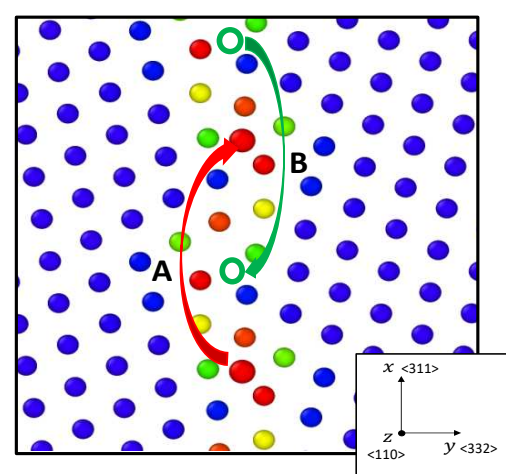

(c)

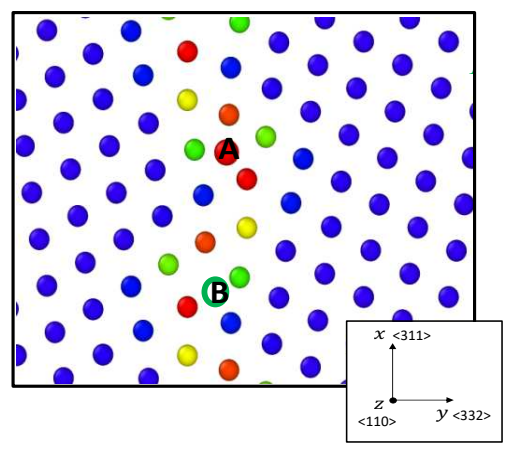

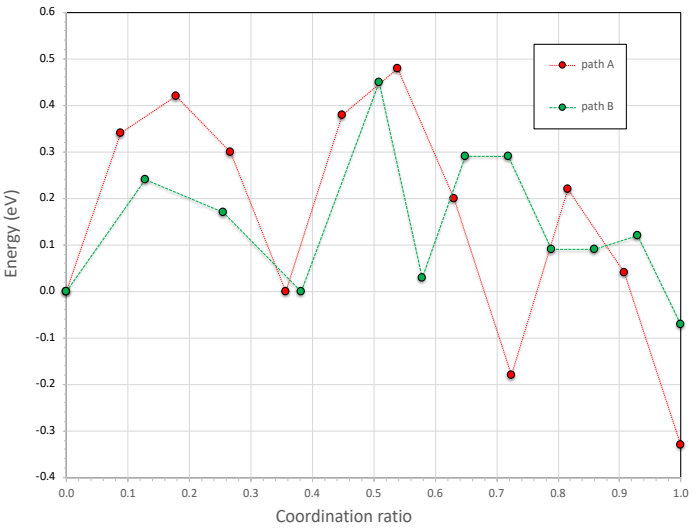
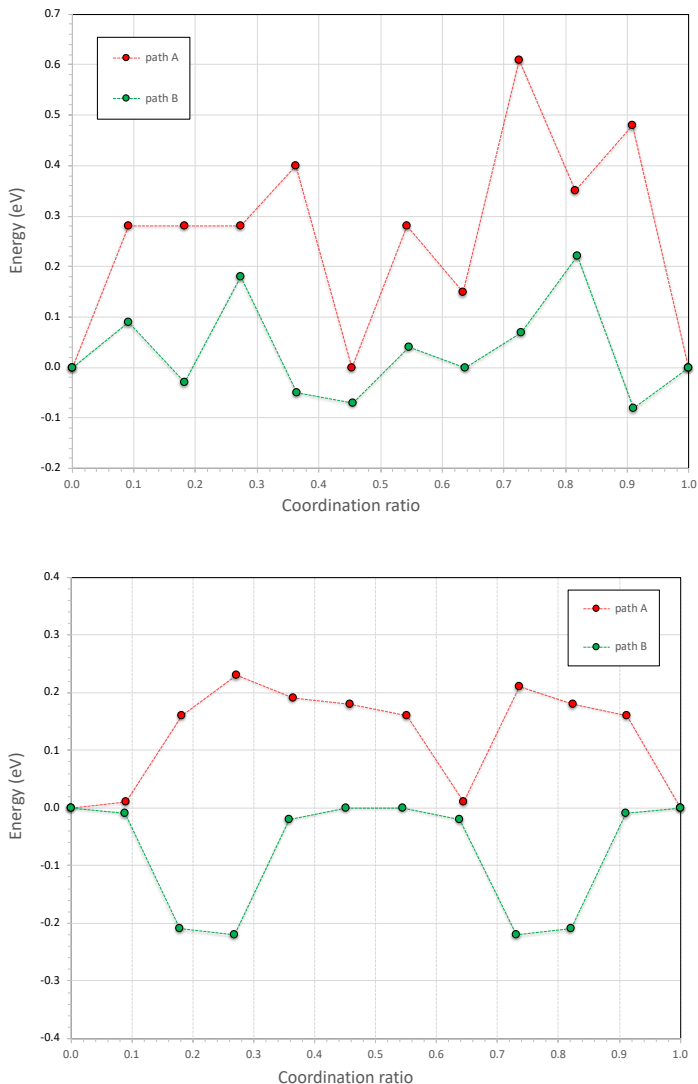

Figure 6 : Energy barriers and minimum energy path between the known initial and final states for the GB $\Sigma 11-\{332\}$. (a) diffusion paths perpendicular to the grain boundary $y=<332>$. (b) and (c) diffusion paths along the grain boundary respectively for $\mathrm{x}=<311>$ and $\mathrm{z}=<110>$. A is the path between the most stable segregation positions and the bulk. $\mathrm{B}$ is the path between the highest volume positions and the bulk. 


\section{Discussion}

The implications of grain-boundary on hydrogen embrittlement is one key understanding to extend the material performance in an aggressive environment. The design of new materials and alloys requires to improve the fundamental interaction of hydrogen with structural features of grain-boundaries. Before any analysis of the intergranular fracture is necessary to understand the mobility of hydrogen in relation to the microstructural features. Commonly as any solutes, the first step is to focus on the segregation process. The general approach of the segregation is based on a differentiation between an insertion site in GB and the one in lattice (octahedral in fcc alloys) in terms of energy and volume $e^{59,60,66,93}$. The domain of the insertion is defined as a volume with specific geometry using space tessellation or Voronoi description. Zhou et al. ${ }^{57}$ recently argued that the local volume of the site can be analyzed using only five polyhedrons. Our present investigations clearly illustrate the limitation of this description where we observed a large diversity of geometric forms (see complementary data) especially for grain boundaries with the highest excess volume. According to a large set of data, the segregation energy cannot be directly correlated with the variation of the volume (figure $3 \mathrm{~d}$ ) without considering the asymmetry of some sites (see figure $3 \mathrm{c}$ site 1) and their distortion (deviatoric part of local strain tensor) during the incorporation of the solute. Another aspect needs to be integrated into this analysis in relation to the impact of hydrogen on the atomic configuration around the insertion site, which depends on the distribution of the different sites along the GB. The first aspect can be considered as a short-range interaction while the second one which corresponds to a long-range distortion according to the length scale larger than the size of the site and the in inhomogeneity of the distribution of these sites. According to the thermodynamic approach, Larche et al. ${ }^{85}$ and Kirchheim et al. ${ }^{86}$ highlighted the possible contribution of the hydrostatic and the deviatoric strain energy on the chemical potential. More recently, the contribution of defects and elastic anisotropy have been questioned on the solubility and diffusivity in nickel ${ }^{16}$. The contribution of shear elastic energy far from any defect stays less common feature related in the literature but a few works show clearly the significance of that one ${ }^{69-71,73,87}$. The shear or deviatoric elastic strain energy $E_{e l}^{d}$ can be mostly near the dislocation core and have a large contribution to the trapping energy $^{70,71}$. In the present work, the respective contribution on elastic strain energy of hydrostatic $E_{e l}^{m}$ and deviatoric $E_{e l}^{d}$ strains in grain boundary (figures $4 \mathrm{~b}$ and $4 \mathrm{~d}$ ) reveals a significant influence of shear strain in domain II for higher hydrogen atomic volume $\left(\mathrm{V}_{\mathrm{H}}>6.6 \AA^{3}\right)$. Additionally, no clear correlation can be obtained in this regime between segregation energy and hydrogen atomic volume (figure $3 \mathrm{~d}$ ). The second fundamental aspect emerges in a contribution of a long-range elastic distortion $E_{\mathrm{el}}^{\mathrm{LR}}$ to segregation energy as illustrated in figure 5. The importance of this contribution was appropriately illustrated for some specific sites (figure 5) where the amplitude of this energy offers the opportunity to explain the discrepancy observed between some 
experimental data and linear relation between the segregation energy $E_{\text {seg }}$ and hydrogen energy $\mathrm{E}_{\mathrm{H}}$ (figure 4a).

Consequently, the segregation energy can be formulated as a function of the different contributions $\left(\mathrm{E}_{\mathrm{el}}^{\mathrm{d}}, \mathrm{E}_{\mathrm{el}}^{\mathrm{m}}, \mathrm{E}_{\mathrm{el}}^{\mathrm{LR}}, \mathrm{E}_{\text {chem }}, \mathrm{E}_{\mathrm{octa}}^{\text {ins }}\right)$ :

$\mathrm{E}_{s e g}=\left[\frac{\mathrm{V}_{o c t}}{\Omega_{N i}}\right] E_{H}-\mathrm{E}_{\text {octa }}^{i n s}+\mathrm{E}_{\mathrm{el}}^{\mathrm{LR}}$ with $E_{H}=E_{e l}^{m}+E_{e l}^{d}+\mathrm{E}_{c h e m}$

The chemical component of energy, $\mathrm{E}_{\text {chem }}$ evolves between $-0.653 \mathrm{eV}$ to $-0.85 \mathrm{eV}$, the short range elastic energy $E_{e l}^{m}+E_{e l}^{d}$ is evaluated between $0.082 \mathrm{eV}$ to $0.286 \mathrm{eV}$, the long range internal stresses can be reach a value of $0.44 \mathrm{eV}$ and the insertion energy for octahedral site $E_{\text {oct }}^{\text {ins }}$ is equal to $0.1775 \mathrm{eV}$.

The hydrogen enrichment at the different grain boundaries studied in the present work can be described with a modified analytical Langmuir-McLean segregation isotherm (LML). Instead of initial approach ${ }^{88}$, it was recently answered ${ }^{89}$ the importance to consider the variability of the segregation energy of one grain boundary ${ }^{90}$. Consequently, for each grain boundary, we have determined the number of sites for each segregation energy for one representative motif (see figure $7 \mathrm{a}$ as an example for $\Sigma 11-\{332\})$. Considering the segregation energy density of states, $\rho\left(E_{\text {seg, }, i}\right)$ for each GBs, we observed that $\rho$ is maximum for low segregation energy in $\Sigma 3-\{111\}$ (Figure $7 b$ ). In opposite, $\rho$ is maximum for the highest segregation energy for $\Sigma 5-\{310\}$ (Figure $7 b)$. The segregation energy corresponding to the maximum of $\rho$ follows the relation : $\mathrm{E}_{\mathrm{seg}}(\Sigma 3-$ $\{111\})>\mathrm{E}_{\text {seg }}(\Sigma 11-\{311\})>\mathrm{E}_{\text {seg }}(\Sigma 11-\{332\})>\mathrm{E}_{\text {seg }}(\Sigma 5-\{310\})$. This order is similar to the one of excess volume of GBs and allows to conclude that $\Sigma 5-\{310\}$ presents a higher capability to segregation than $(\Sigma 11-\{332\})$ although the number of sites is lower to the one of $\Sigma 11-\{332\}$. 

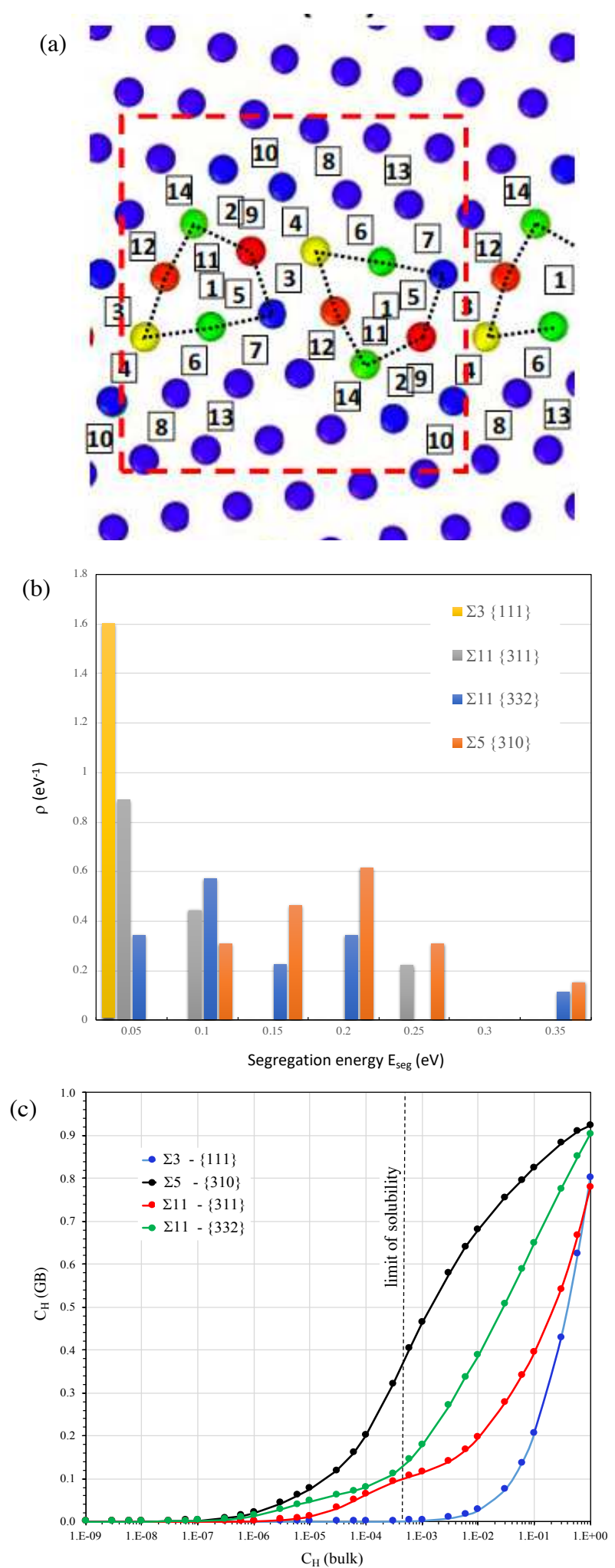

Figure 7 : (a) The unit cell for GB $\Sigma 11-\{332\}$ with position details ((31 Ni and 28 sites for hydrogen segregation, 14 sites with different segregation energies). (b) the segregation energy density of states ( $\rho$ ) as a function of absolute value of segregation energy Eseg for four GBs. (c) the evolution of hydrogen concentration segregated on GBs is represented as a function with the bulk concentration. 
According to the Langmuir-McLean segregation isotherm model without solutes interactions ${ }^{66,67}$, it is possible to establish a relationship between the segregation concentration and the bulk concentration in a binary system. The expression is given in:

$C_{G B}=\sum_{i=1}^{n} C_{\max , i} \frac{1}{1+\frac{1-C_{\text {bulk }}}{C_{\text {bulk }}} \exp \left[\frac{E_{\text {seg }, i}}{k_{B} T}\right]}$

$C_{s e g, i}=C_{H s e g, i} / C_{N i}$ is the molar fraction of segregation on site i, $C_{b u l k}=C_{H} / C_{N i}$ is the molar fraction in the nickel bulk. Hydrogen atoms occupy the octahedral site in the nickel bulk, the unit cell of fcc structure gives the maximum saturation $C_{o c t} / C_{N i}=1$. The saturation of segregation $C_{\text {max, }}$ of the boundary on a site $\mathrm{i}$ depends on the number of the sites with the same segregation energy $\mathrm{E}_{\mathrm{seg}, \mathrm{i}}$. As illustrated in figure $7 \mathrm{a}$ for $\Sigma 11-\{332\} \mathrm{n}=14$ sites can be distinguished. Considering the temperature of $300 \mathrm{~K}$, the evolution of hydrogen concentration segregated on GBs is represented as a function of the bulk concentration (Figure 7c). The picture confirms the analysis in terms of the segregation energy density of states. The segregation sites in the high energy GBs such as $\Sigma 11-\{332\}$ and $\Sigma 5-\{310\}$ start to be occupied by hydrogen atoms at a very low bulk concentration $\left(C_{b u l k} \sim 10^{-7} \mathrm{H} / \mathrm{Ni}\right)$. The GB $\Sigma 11-\{311\}$ begins the segregation around $C_{b u l k}=10^{-6} \mathrm{H} / \mathrm{Ni}$ while the GB $\Sigma 3-\{111\}$ initiates the segregation around $C b u l k=10^{-3} \mathrm{H} / \mathrm{Ni}$. The segregation is first initiates at the most stable positions, and then the less steady positions commence to segregate hydrogen atoms when the first position reaches saturation (70\% - 80\% occupation). It has been reported that high local concentrations can act as a seed for local hydrides in nickel ${ }^{91,92}$ because of the attractive $\mathrm{H}-\mathrm{H}$ interactions. Moreover, EP/TDS experimental data indicates that the limit of hydrogen solubility in nickel single crystal is around $C_{b u l k}=5 \times 10^{-4} \mathrm{H} / \mathrm{Ni}(\sim 7.5$ wppm at $300 \mathrm{~K})$. Consequently, we compared thus the segregation in different GBs within the range of the bulk concentration between $10^{-7}$ and $5 \times 10^{-4} \mathrm{H} / \mathrm{Ni}$. Hydrogen segregation is stronger in $\Sigma 5-\{310\}$ than in $\Sigma 11-\{332\}$, the segregation concentration is dominated by the most stable segregation position of which $\mathrm{E}_{\mathrm{seg}, 1}(\Sigma 5-\{310\})=-0.3252 \mathrm{eV}<\mathrm{E}_{\mathrm{seg}, 1}(\Sigma 11-\{332\})=-0.3188 \mathrm{eV}$. Additionally, the GB $\Sigma 3-\{111\}$ acts as the nickel bulk with no significant segregation at all.

Once hydrogen atom is localized in a GB, it is now appropriated to ask now how the atom will move or remain trapped using the evaluation of the migration energies.

The hydrogen atom in all GBs $(\Sigma 3-\{111\}, \Sigma 11-\{311\}, \Sigma 5-\{310\}$ and $\Sigma 11-\{332\})$ can easily move from the nickel bulk to the $\mathrm{GB}$ core: the forward energy $\mathrm{E}_{\mathrm{m}}^{\mathrm{GB}, \mathrm{Y}}=\mathrm{E}_{\mathrm{m}}^{\mathrm{F}} \approx 0.47 \pm 0.01 \mathrm{eV}$ is quite similar to the migration energy in the bulk $\mathrm{E}_{\mathrm{m}}^{\mathrm{bulk}}=\mathrm{E}_{\mathrm{m}}^{0-\mathrm{T}}=0.47 \mathrm{eV}$. GBs such as $\Sigma 11-\{311\}, \Sigma 5-$ $\{310\}$ and $\Sigma 11-\{332\}$ prevent the hydrogen atom from moving from the GB core to the bulk (backward energy $\mathrm{E}_{\mathrm{m}}^{\mathrm{GB}, \mathrm{Y}}=\mathrm{E}_{\mathrm{m}}^{\mathrm{B}}>0.47 \mathrm{eV}$ ), however $\Sigma 3-\{111\}$ GB act if the bulk had little influence on this direction $\left(\mathrm{E}_{\mathrm{m}}^{\mathrm{GB}, \mathrm{Y}}=\mathrm{E}_{\mathrm{m}}^{\mathrm{B}}=0.46 \mathrm{eV}\right)$. The energy barrier is able to provide an 
estimation for the ratio of the diffusion coefficient in comparison to the diffusion in the perfect nickel bulk as it was previously proposed by Di Stefano et al. ${ }^{52}$ :

$$
\frac{D_{G B}}{D_{\text {bulk }}}=\exp \left(-\frac{E_{m}^{G B, i}-E_{m}^{\text {bulk }}}{k_{B} T}\right) \text { with } i \in\{X, Y, Z\}
$$

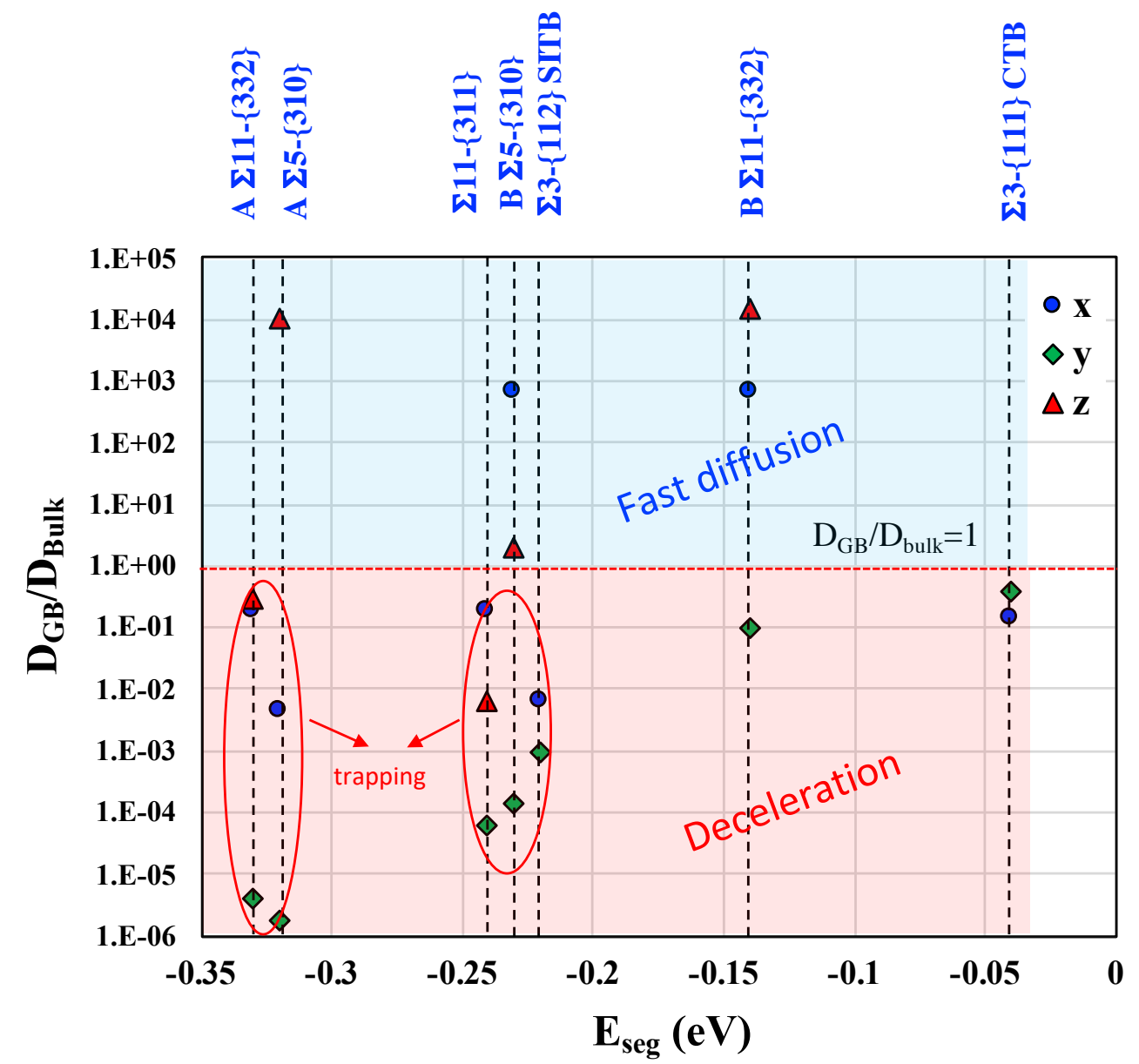

Figure 8 : The diffusion coefficient ratio as a function of the hydrogen segregation energy following $\mathrm{x}, \mathrm{y}$ and $\mathrm{z}$ directions for different GBs.

Two situations can be considered when the backward energy is higher than the one in bulk. The first one corresponds to the segregation of hydrogen to an easy migration path along the grain boundary (directions $\mathrm{X}$ and/or $\mathrm{Z}$ ) where the $\mathrm{E}_{\mathrm{m}}^{\mathrm{GB}, \mathrm{X} \text { or Z }}$ is lower than in the bulk and consequently $\frac{D_{G B}}{D_{b u l k}}>1$. This situation is commonly named a short circuit of diffusion. The second case is a situation where all the migration energies along GB are higher than in the bulk and consequently $\frac{\mathrm{D}_{\mathrm{GB}}}{\mathrm{D}_{\mathrm{bulk}}}<1$. This situation is defined as a trapping configuration. Figure 8 illustrates a distinction (bulk red line) between the accelerated diffusion and the trapping in segregation sites type A 
(most stable segregation sites) and B (highest volume sites) for the different GBs studied. High energy/excess volume GBs ( $\Sigma 11-\{332\}$ and $\Sigma 5-\{310\})$ have one or two accelerated diffusion path(s) where the hydrogen atom moves $10^{3} \sim 10^{4}$ faster than the diffusion in the nickel bulk. These results are confirmed by our experimental data where for both GBs the hydrogen content in bicrystals is lower than in single crystal for the same orientation (Figure 1c and 1d). Indeed, $\Sigma 11$ $\{332\}$ is a GB that have more segregation sites in the GB core with two accelerated diffusion paths, and none of these segregation sites has the trapping behavior (Figure 8). While GB $\Sigma 5$ $\{310\}$ has only one accelerated diffusion path and the deepest segregation site in this GB is a trapping position for the hydrogen atoms (Figure 8). $\Sigma 11-\{311\}$ GB do not present any accelerating diffusion paths but only trapping sites in the GB core. Moreover, no significant difference is observed in terms of hydrogen content in single and by-crystals (Figure 1d). These results allow to conclude that this GB is not a fast diffusion path as $\Sigma 3-\{111\}$. Similar results in GBs $\Sigma 11-\{311\}$ and $\Sigma 3-\{111\}$ have been reported by Du et al. ${ }^{63}$ in $\gamma$-Fe based on their DFT calculations. To conclude, based on a combined analysis of the segregation energy and migration energy of the different potential sites of hydrogen in one GB, it is possible to define one GB as a short circuit of diffusion or trapping location for hydrogen. In perspective, we can enlarge this analysis for a large number of GB configurations considering the similarity in terms of $\mathrm{E}_{\mathrm{m}}^{\mathrm{GB}, \mathrm{i}}$ and $E_{H}$ using atomistic calculation and machine learning ${ }^{89}$.

\section{Conclusion}

We developed a methodology based on the confrontation between experimental hydrogen charging and atomistic modeling to elucidate the competition between hydrogen trapping and short circuit of diffusion along grain boundaries.

Using thermal desorption spectroscopy and electrochemical permeation testing, we measured the diffusivity of hydrogen and trapping energy in single and poly crystals of nickel on a large variety of configurations. Our experimental results are challenged by our atomistic simulations to provide a straightforward understanding of the apparently ambiguous and antagonist effects of grain boundaries between the trapping process and the fast diffusion path. The origin of the acceleration of the diffusivity along grain boundaries is observed when the excess volume increases, which is clarified in terms of migration energies and the distribution of segregation energies. The fast diffusion of hydrogen observed in some grain boundary configurations corresponds to high segregation energy sites and different paths along inter-connected sites of low migration energy. In opposite, the trapping process occurs in grain boundaries of high segregation energy sites and high migration energies. We underline the importance of the elastic energy to global hydrogen incorporation energy in one specific site of grain boundaries. Due to the complex structure of the grain boundary, the distribution of the segregation energy depends not only on the hydrostatic 
strain energy but also on the deviatoric elastic energy and the long-range elastic distortion which can predominate far from GB. The present work can be extended to a large variety of grain boundaries to allow a more generic relationship between elastic, segregation and migration energies.

\section{Methods}

Material design and structural characterization -The different nickel samples investigated were high purity single crystals (99.999\% purity), bi-crystals (99.95\% purity) and polycrystals/nanocrystals (99.99\% purity). Nickel single crystals with three different crystallographic orientations $<001>,<110>$ and $<111>$ were provided by Goodfellow. Samples are cylindrical with an $18 \mathrm{~mm}$ diameter for nickel $<001>$ and $11 \mathrm{~mm}$ for nickel $<110>$ and $<111>$. They were obtained by Bridgman-Stockbarger method with an accuracy of $\pm 3^{\circ}$. Nickel bi-crystals were made in Laboratory (provided by professor L. Priester (ICMPE) and developed in Mines St-Etienne School). The material for solidification was electrolytic nickel which was remelted under vacuum. The bicrystals were grown from a seed in an argon atmosphere using the horizontal boat method (Chalmers method) of a final length of $14.4 \mathrm{~cm}$ at a rate of about $3 \mathrm{~mm}$ per hour. The impurity contents are given lower than 0.05 wppm, the sulfur content could be enough to promote intergranular segregation ${ }^{94}$. All samples were then carried out a desulfurization heat treatment at $550^{\circ} \mathrm{C}$ for 10 hours in flowing hydrogen. Polycrystalline nickel with micrometric grain sizes between $18 \mu \mathrm{m}$ and $200 \mu \mathrm{m}$ was controlled by thermo-mechanical processing at constant annealing temperature for a predefined time in a controlled atmosphere on cold-drawn round nickel rods provided by Goodfellow ${ }^{95}$. The nanometric sizes from 20 to $120 \mathrm{~nm}$ were synthesized by electrodeposition using a conventional additive-free sulfamate bath according to the deposition parameters and conditions described previously ${ }^{96}$.

All the microstructures were characterized using Electronic Back Scatter Diffraction (EBSD) and Transmission Electronic Microscopy (TEM). EBSD analyses using an EDAX/ TSL OIM system coupled to an FEI Quanta 200 ESEM- FEG scanning electron microscope were used to characterize the crystallographic orientation (texture, surface orientation) and grain boundaries character (misorientation, coincidence site lattice) ${ }^{28}$. Dislocation densities of each sample were investigated using a JEOL JEM 2011 electron microscope operating at $200 \mathrm{kV}$. Foils for transmission electron microscopy (TEM) were thinned in a double twin-jet electro-polisher using electrolyte under the conditions described previously ${ }^{97}$. An estimation of the dislocation density of the as-received material gave an average value of less than $10^{-10} \mathrm{~m}^{2}$.

Charging hydrogen and permeation conditions -The electrochemical permeation (EP) test and thermal desorption spectroscopy (TDS) were used to characterize macroscopically the diffusivity 
and concentration of hydrogen for different conditions. The EP technique introduced by Devanathan and Stachurski ${ }^{99}$ is the main technique used to detect the mechanisms of diffusion and trapping of hydrogen in different microstructures ${ }^{15,16,28}$. This technique is composed of two cells separated by a membrane with an exposed surface in contact with an electrolytic solution. The charging side (entry side) of the EP was galvanostatically polarized at a constant cathodic charging current density (5 to $100 \mathrm{~mA} / \mathrm{cm}^{2}$ ) in $0.1 \mathrm{M} \mathrm{NaOH}$. The detection side (exit side) of the EP was maintained with a constant anodic potential of $\sim 630 \mathrm{mV} / \mathrm{SSE}$ in $0.1 \mathrm{M} \mathrm{NaOH}$, and the hydrogen flux (current density) at the detection side was recorded to study the transport of hydrogen through the membrane. The temperature was maintained at $300 \mathrm{~K}$ and both solutions were continuously deaerated under argon flux at 1.4 bar. Before the permeation test, both surfaces of the sample were prepared by mechanical polishing up to grade $4000 \mathrm{SiC}$; the final thickness of the sample was about $200 \pm 20 \mu \mathrm{m}$.

To quantify the hydrogen concentration and the maximum solubility in nickel, we used Thermal Desorption Spectroscopy (TDS) ${ }^{15,28}$. These analyses were performed with a Jobin Yvon Horiba EMGA-621W hydrogen analyzer composed of an impulsion furnace system coupled with a thermal conductivity detector. The procedure used consists involved measuring the hydrogen concentration in the pre-charged samples $(0.1 \mathrm{M} \mathrm{NaOH}$ at $298 \mathrm{~K}$ under galvanostatic polarization $-10 \mathrm{~mA} / \mathrm{cm}^{2}$ with different time) by fusion. For this purpose, after hydrogen charging, the specimen (dimension $8 \times 4 \times 0.3 \mathrm{~mm}^{3}$ ) were mechanically polished with $5 \mu \mathrm{m} \mathrm{SiC} \mathrm{grinding} \mathrm{paper,}$ and then cleaned in acetone before introduced into the furnace, where they were instantly heated to $2000^{\circ} \mathrm{C}$ and maintained at this temperature for 75 seconds. The desorbed hydrogen was then detected and analyzed by gaseous Catharometry. The recorded curve corresponds to the amount of hydrogen detected as a function of time. The average concentration of hydrogen in the sample was estimated by measuring the area under the curve.

Atomistic simulation - The atomistic investigation following the works of Hallil et al. ${ }^{47,61}$ was carried out using LAMMPS code (Large-scale Atomic/Molecular Massively Parallel Simulator) for MD simulations at $0 \mathrm{~K}$, or more precisely, molecular statics (MS). The atomistic simulations based on the relaxed configuration of atoms are found using the minimization of the total energy with an appropriate interatomic potential at $0 \mathrm{~K}$ and $0 \mathrm{~Pa}$. For the $\mathrm{H}-\mathrm{Ni}$ system, the interatomic potential was established by the embedded-atom method $(\mathrm{EAM})^{54}$. The energy $\mathrm{E}_{\mathrm{i}}$ of atom i $(\mathrm{H}$ or Ni) in the EAM method is given by the following equation:

$E_{i}=F_{\alpha} \sum_{i \neq j} \rho_{\beta}\left(r_{i j}\right)+\frac{1}{2} \sum_{i \neq j} \varphi_{\beta}\left(r_{i j}\right)$

where $\mathrm{F}_{\alpha}$ is the embedding energy function, $\rho$ is the partial electron density contribution, $r_{i j}$ denotes the distance between atom $\mathrm{i}$ and $\mathrm{j}, \varphi$ is the pair potential, $\alpha$ and $\beta$ are the element types of atom $\mathrm{i}$ and j. For a grain boundary in a bi-crystal system, the construction of grain boundary is built by finding 
the most optimal configuration. An example of a simulation cell for the grain boundary $\Sigma 5$ $36^{\circ} 87<100>\{310\}$ is illustrated in figure A1.

(a)

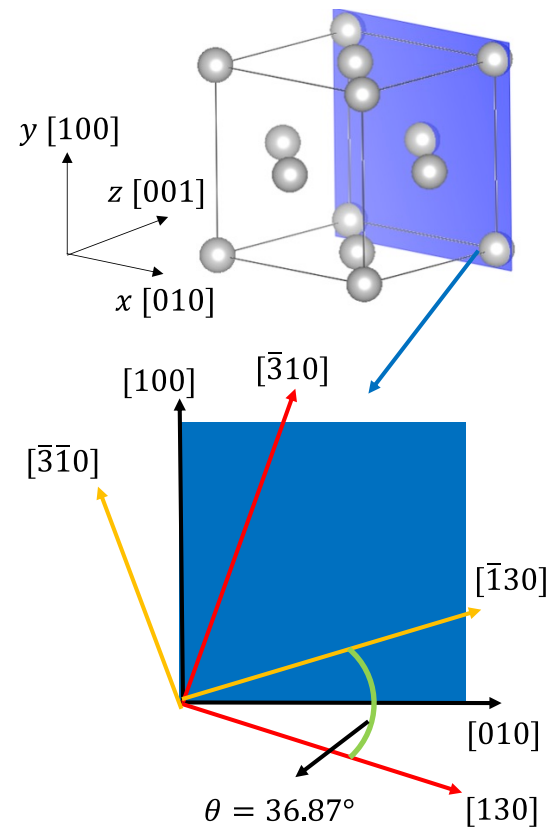

(b)

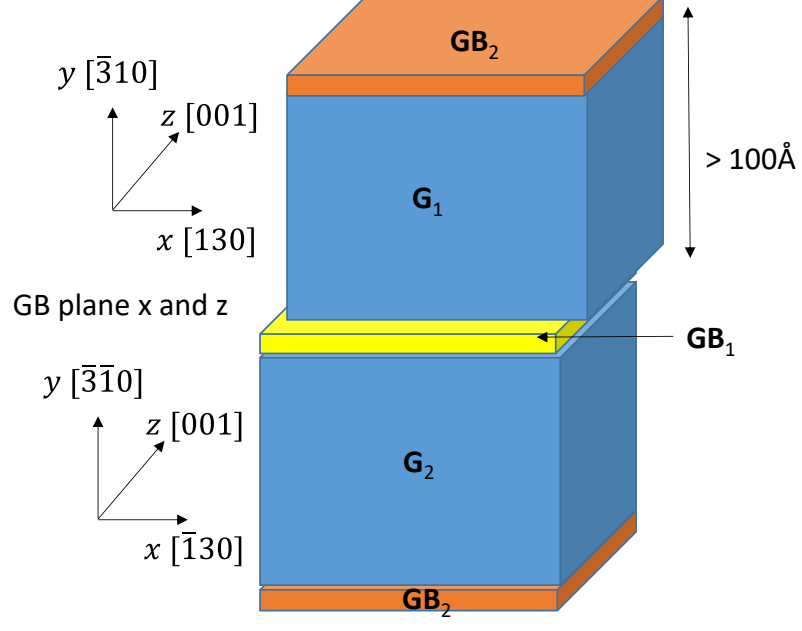

Figure A1- (a) GB $\Sigma 5-36^{\circ} 87<001>\{310\}$ plane for nickel. (b) Schematic of a bi-crystal simulation cell.

$\theta$ is the misorientation angle between two identical nickel crystals around the symmetric tilt axis along the grain boundary plane. The direction along the GB plane is designated by the tilt z-axis [001] in the simulation cell and common for both grains. Each GB simulation cell contains two-grain lattices which are characterized by two distinct crystallographic orientations in $\mathrm{x}$ and $\mathrm{y}$ directions. The GB simulation cells are considered in 3D periodic boundary conditions, this representation provides existence of two GBs in each simulation cell: one in the middle of the simulation cell and on another counting for a mirror image in the bound parts of the simulation cell. The separation distance between each GB is chosen to be large enough so that there will be no energetic interaction between two GB interfaces. A rigid body translation parallel to the GB plane has been applied following $\mathrm{x}$ and $\mathrm{z}$-axis, all translational vectors are in a primitive cell of the displacement shift complete (DSC) lattice ${ }^{60}$ and the lattice spacing in the planar directions of each grain is kept constant. The translation of one grain relative to the other yields to a re-arrangement of atoms at the GB plane. After testing hundreds of configurations, the one with the minimum energy at the grain boundary is obtained and the excess volume could be calculated using Voronoi tessellation method implemented in LAMMPS code.

Since the GB structure is well defined, we can insert an atom of hydrogen into the GB. The initial position of the hydrogen atom is at the vicinity of a nickel atom. Then the energy minimization occurs and the hydrogen atom will find its stable position using atomic simulation analysis 
OVITO software. After determining several stable segregation sites in the core region, we focused on the diffusion paths among these different segregation sites using the nudged elastic band method (NEB) [100]. This method is used to detect saddle points and minimum energy paths (MEP) between the known initial and final states. Transition states of diffusion paths (referred to as images) identify the lowest possible energy while maintaining equal spacing to neighboring images. Once the images have converged sufficiently to the MEP, the image at the highest energy point is allowed to climb uphill along the MEP until it reaches the transition state enabling thus the transition geometry and energy to be accurately defined from the NEB method [101]. We have investigated the MEP and the energy barrier between the most stable segregation positions and the highest Voronoi volume positions (the volume occupied by the hydrogen atom) using LAMMPS code with NEB package. The GB energy is computed as the difference between the total energy of the relaxed GB atoms and the bulk energy in the whole system in GB plane. For a number of atoms $N_{a t}$ in the calculation, the grain boundary energy $E_{G B}$ is given as:

$$
E_{G B}=\frac{1}{2}\left[E_{G B}^{N i}\left(N_{a t}\right)-E_{B u l k}^{N i}\left(N_{a t}\right)\right] / A_{0}
$$

Where $E_{G B}^{N i}\left(N_{a t}\right)$ is the total energy of the relaxed GB, $E_{B u l k}^{N i}\left(N_{a t}\right)$ is the total bulk energy, $A_{0}$ is the area of the GB plane. $\Delta V_{G B}$ the excess volume of a GB can be accessed with (volume variation per unit of GB area):

$\Delta V_{G B}=\frac{1}{2}\left[V_{G B}^{T o t}\left(N_{a t}\right)-V_{B u l k}^{T o t}\left(N_{a t}\right)\right] / A_{0}$

where $V_{G B}^{T o t}\left(N_{a t}\right)$ is the volume of GB, $V_{B u l k}^{T o t}\left(N_{a t}\right)$ is the volume of bulk.

When the stable configuration of GBs has been established, we started to insert the hydrogen atom in different locations in the GBs. The insertion energy (in some publications called also the adsorption energy) of a hydrogen atom in the nickel lattice $E_{H}^{I n s}$ is given in (eq. A3):

$$
E_{H}^{I n s}=E_{N i+H}^{T o t}-E_{N i}^{T o t}-\frac{1}{2} E_{H_{2}}
$$

$E_{N i+H}^{T o t}$ is the total energy of nickel lattice with a hydrogen atom, $E_{N i}^{T o t}$ is the total energy of nickel lattice without a hydrogen atom, $E_{\mathrm{H}_{2}}$ is the chemical potential of the molecular hydrogen and the binding energy of one hydrogen atom can be obtained by the EAM potential in vacuum ${ }^{98}\left(\frac{1}{2} E_{\mathrm{H}_{2}}=\right.$ $-2.36947 \mathrm{eV})$. This calculation has indicated that the octahedral site $\left(E_{O c t}^{I n s}=0.1775 \mathrm{eV}\right)$ is more 
stable than the tetrahedral site $\left(E_{T e t}^{I n s}=0.586 \mathrm{eV}\right)$. Similar results of EAM have been reported by Huang et al. ${ }^{55}$. Thus, the segregation energy relative to the octahedral site is written in (eq. A4):

$E_{H}^{s e g}=\left(E_{G B+H}^{T o t}-E_{G B}^{T o t}\right)-\left(E_{N i+H}^{o c t}-E_{N i}^{o c t}\right)$

$E_{G B+H}^{T o t}$ is the total energy of GB with a hydrogen atom, $E_{G B}^{T o t}$ is the total energy of the GB and $E_{N i+H}^{O C t}$ is the total energy of the nickel lattice with a hydrogen atom at the octahedral site.

\section{References}

[1] Martin F., Feaugas X., Oudriss A., Tanguy D., Briottet L., Kittel J. State of Hydrogen in Matter: Fundamental Ad/Absorption, Trapping and Transport Mechanisms, in: C. Blanc, I. Aubert (Eds.), Mechanics - Microstructure - Corrosion Coupling, Elsevier, chapter 8, 171-197 (2019).

[2] Ashby M. F., Hirth J.P. Perspectives in Hydrogen in Metals: Collected Papers on the Effect of Hydrogen on the Properties of Metals and Alloys, Elsevier, (1986).

[3] Gangloff, B.P. Somerday (Eds.) Gaseous Hydrogen Embrittlement of Materials in Energy Technologies: Mechanisms, Modelling and Future Developments, Woodhead Publishing LtD, Cambridge, 247-285 (2012).

[4] Robertson I.M. Hydrogen Embrittlement Understood, Metall. Mat. Trans. A 46, 2323-2341 (2015).

[5] Nagumo M. Fundamentals of hydrogen embrittlement, Springer, Singapore (2016).

[6] Feaugas, X., Delafosse, D., Hydrogen and Crystal Defects Interactions: Effects on Plasticity and Fracture, in: Mechanics - Microstructure - Corrosion Coupling. Elsevier Publisher chapter 9, 199222. (2019).

[7] Kimura A., Birnbaum H.K. Hydrogen induced grain boundary fracture in high purity nickel and its alloys-Enhanced hydrogen diffusion along grain boundaries. Acta Metallurgica 36, 757-766 (1988).

[8] Martin, M.L., Somerday, B.P., Ritchie, R.O., Sofronis, P., Robertson, I.M. Hydrogen-induced intergranular failure in nickel revisited. Acta Materialia 60 (6-7) 2739-2745 (2012).

[9] Oudriss A., Bouhattate J., Savall C., Creus J., Feaugas X., Martin F.A., Laghoutaris P., Chêne J. On the Implication of Hydrogen on Inter-granular Fracture. Procedia Materials Science 3, 2030-2034 (2014).

[10] Seita M., Hanson J.P., Gradečak S., Demkowicz M.J. The dual role of coherent twin boundaries in hydrogen embrittlement. Nature Com. 6, 6164 (2015).

[11] Harris, Z.D., Lawrence, S.K., Medlin, D.L., Guetard, G., Burns, J.T., Somerday, B.P. Elucidating the contribution of mobile hydrogen-deformation interactions to hydrogen-induced intergranular cracking in polycrystalline nickel. Acta Materialia 158, 180-192 (2018).

[12] Bertsch K.M., Wang S., Nagao A., Robertson I.M., Hydrogen-induced compatibility constraints across grain boundaries drive intergranular failure of Ni, Materials Science Eng. A 760 58-67 (2019).

[13] Lynch S. A review of underlying reasons for intergranular cracking for a variety of failure modes and materials and examples of case histories. Engineering Failure Analysis 100, 329-350 (2019).

[14] Koyama M., Rohwerder M., Tasanc C.C., Bashir A., Akiyama E., Takaif K., Raabeg D. and Tsuzakia $\mathrm{K}$. Recent progress in microstructural hydrogen mapping in steels: quantification, kinetic analysis, and multi-scale characterization. Mater. Sci. and Tech. 33, 1481-1496 (2017).

[15] Oudriss A., Martin F., Feaugas X. Experimental Techniques for Dosage and Detection of Hydrogen, Editor(s): Christine Blanc, Isabelle Aubert, Mechanics - Microstructure - Corrosion Coupling, Elsevier, chapter 11, 245-268 (2019).

[16] Li J., Oudriss A., Metsue A., Bouhattate J., Feaugas X. Anisotropy of hydrogen diffusion in nickel single crystals: the effects of self-stress and hydrogen concentration on diffusion. Scientific Rep. 7, 45041 (2017). 
[17] Hachet G., Metsue A., Oudriss A., Feaugas X. Influence of hydrogen on the elastic properties of nickel single crystal: A numerical and experimental investigation. Acta Mater. 148, 280-288 (2018).

[18] Kirchheim R. Somerday B., Sofronis P. Chemomechanical effects on the separation of interfaces occurring during fracture with emphasis on the hydrogen-iron and hydrogen-nickel system. Acta Materialia 99 87-98 (2015).

[19] Alvaro A., Thue Jensen I., Kheradman N. , C, Løvvik O.M., Olden V. Int. J. of Hydrogen 40, 1689216900 (2015).

[20] Chen, J., Dongare, A.M. Role of grain boundary character on oxygen and hydrogen segregationinduced embrittlement in polycrystalline Ni. J. Mater Sci. 52, 30 (2017)

[21] Tehranchi A., Curtin W.A. Atomistic study of hydrogen embrittlement of grain boundaries in nickel: I. Fracture, J. Mech. and Phys. of sol. 101, 150-165 (2017).

[22] Hea, S. Eckera W., Pippanc R., Razumovskiy V.I., Hydrogen-enhanced decohesion mechanism of the special $\Sigma 5(012)[100]$ grain boundary in Ni with Mo and C solutes, Comp. Mater. Science 167 100-110 (2019).

[23] Kuhr B. Farkas D., Robertson I.M. Atomistic studies of hydrogen effects on grain boundary structure and deformation response in FCC Ni. Comp. Mater. Science 122 92-101 (2016).

[24] Louthan MR, Donovan JA, Caskey GR. Hydrogen diffusion and trapping in nickel. Acta Metall. 23, 745 (1975).

[25] Kirchheim R, Kownacka I, Filipek SM. Hydrogen segregation at grain boundaries in nanocrystalline nickel. Scripta Metall. 28, 1229 (1993).

[26] Brass AM, Chanfreau A. Accelerated diffusion of hydrogen along grain boundaries in nickel. Acta Mater. 44, 3823 (1996).

[27] Oudriss A., Creus J., Bouhattate J., Savall C., Peraudeau B., Feaugas X. The diffusion and trapping of hydrogen along the grain boundaries in polycrystalline nickel. Scripta Mater. 66, 37-40 (2012).

[28] Oudriss A., Creus J., Bouhattate J., Conforto E., Berziou C., Savall C., Feaugas X. Grain size and grain-boundary effects on diffusion and trapping of hydrogen in pure nickel. Acta Materialia 60 6814-6828 (2012).

[29] Oudriss A., Le Guernic S., Wang Z., Osman Hoch B., Bouhattate J., Conforto E., Zhu Z., Li D.S., Feaugas X. Meso-scale anisotropic hydrogen segregation near grain-boundaries in polycrystalline nickel characterized by EBSD/SIMS. Materials Letters 165, 217-222 (2016).

[30] Schober T. and Dieker C. Observation of local hydrogen on nickel surface. Metall. Trans. A 14A 2440-2442 (1983).

[31] Yao J., Cahoon J.R. The Use of Silver Decoration Technique in the Study of Hydrogen Transport in Metallic Materials. Metall. Trans. 21A 603 (1990).

[32] Tanaka T., Kawakami K., Hayashi S., Visualization of deuterium flux and grain boundary diffusion in duplex stainless steel and $\mathrm{Fe}-30 \% \mathrm{Ni}$ alloy, using secondary ion mass spectrometry equipped with a Ga focused ion beam. J. Mater. Sci. 49, 3928 (2014).

[33] Ma Z.X., Xiong X.L., Zhang L.N., Zhang Z.H., Yan Y., Y.J. Su. Experimental study on the diffusion of hydrogen along individual grain boundaries in nickel. Electrochemistry Communications. 92, 2428 (2018).

[34] Tsuru, T. and R.M. Latanision. Grain boundary transport of hydrogen in nickel. Scripta Metall. Mater. 16, 575 (1982).

[35] Fukushima H., Birnbaum H.K., Surface and grain boundary segregation of deuterium in nickel, Acta metall. 32 851-859 (1984).

[36] Ladna B., Birnbaum H.K. SIMS study of hydrogen at the surface and grain boundaries of nickel bicrystals,. Acta metall. 3525352542 (1987).

[37] Karnesky R.A., Norman Jr., Bartelt C., Teslich D., and Kumar M., Imaging and Quantification of Hydrogen Isotope Trapping. Sandia report, sand2012, 8539 (2012).

[38] Dingreville R., Alsoy D., Spearot D.E. A primer on selecting grain boundary sets for comparison of interfacial fracture properties in molecular dynamics simulations. Sci. Reports 78332 (2017).

[39] Tehranchi A., Curtin W., Atomistic study of hydrogen embrittlement of grain boundaries in nickel: I, Fracture, J Mech. Phys. Solids 101 (Supplement C) 150-165 (2017).

[40] Alvaro A., Thue Jensen I., Kheradman N., C, Løvvik O.M., Olden V., Int. J. of Hydrogen 40 1689216900 (2015).

[41] Yu, H., Olsen, J.S., Olden, V., Alvaro, A., He, J., Zhang, Z. Cohesive zone simulation of grain size and misorientation effects on hydrogen embrittlement in nickel. Engineering Failure Analysis, 81, 79-93 (2017).

[42] Tehranchi, A., Curtin, W.A. Atomistic study of hydrogen embrittlement of grain boundaries in nickel: II. Fracture Modelling and Simulation in Materials Science and Engineering. 25 (7), art. no. 075013 (2017). 
[43] Zhou, X.W., Dingreville, R., Karnesky, R.A. Molecular dynamics studies of irradiation effects on hydrogen isotope diffusion through nickel crystals and grain boundaries. Physical Chemistry Chemical Physics, 20 (1) 520-534 (2017).

[44] O'Brien, C.J., Foiles, S.M. Hydrogen segregation to inclined twin grain boundaries in nickel Philosophical Magazine, 96 (26) 2808-2828 (2016).

[45] Kuhr, B., Farkas, D., Robertson, I.M. Atomistic studies of hydrogen effects on grain boundary structure and deformation response in FCC Ni. Comp. Mater. Science, 122, 92-101 (2016).

[46] Tehranchi A., Curtin, W.A., Atomistic study of hydrogen embrittlement of grain boundaries in nickel: I. Fracture, Journal of the Mechanics and Physics of Solids. 101, 150-165 (2017).

[47] Hallil, A., Metsue, A., Oudriss, A., Bouhattate, J., Feaugas, X. Segregation energy of the hydrogen at Ni $\Sigma 3$ grain boundaries: some implications of the atomic volume and the interstitial self-stress. Journal of Materials Science, 53 (7) 5356-5363 (2018).

[48] Moody N.R., Foiles S.M. An atomistic study of the equilibrium segregation of hydrogen to tilt boundaries in nickel. Sandia National Laboratories, Livermore, Mat. Res. Soc. Symp. Proc. 229 (1991).

[49] He S., Ecker W, Pippan R. Razumovskiy V.I. Hydrogen-enhanced decohesion mechanism of the special $\Sigma 5(012)[100]$ grain boundary in $\mathrm{Ni}$ with Mo and C solutes. Computational Materials Science. 167, 100-110 (2019).

[50] Yamaguchi M., Shiga M., Kaburaki H., Energetics of segregation and embrittling potency for nontransition elements in the $\Sigma 5$ (012) symmetrical tilt grain boundary: a first-principles study, J. Phys.: Condens. Matter. 16 (23) 3933 (2004).

[51] Yamaguchi M., Shiga M., Kaburaki H. First-principles study on segregation energy and embrittling potency of hydrogen in ni $\Sigma$ 5(012) tilt grain boundary. J. Phys. Soc. Jpn. 73 (2) 441-449 (2004).

[52] Di Stefano D., Mrovec M., Elsässer C., First-principles investigation of hydrogen trapping and diffusion at grain boundaries in nickel, Acta Mater. 98 (Supplement C) 306-312 (2015)

[53] G. Young, R. Najafabadi, W. Strohmayer, D. Baldey, B. Hamm, J. Harris, J. Sticht, E. Wimmer, An atomistic modeling study of alloying element impurity element, and transmutation products on the cohesion of a nickel ni $\Sigma 5$ \{001\} twist grain boundary. Tech. Report LM-03K047, NASA. URL: (2003).

[54] Angelo J.E., Moody N.R., Baskes M.I., Trapping of hydrogen to lattice defects in nickel. Model. Simul. Mater. Sc. 3 (3) 289 (1995).

[55] Huang S, Chen D., Song J., McDowell D.L., Zhu T., Hydrogen embrittlement of grain boundaries in nickel: an atomistic study, npj, Comp. Mat. 328 (2017).

[56] Chen, J. and Dongare, A.M. Role of grain boundary character on oxygen and hydrogen segregationinduced embrittlement in polycrystalline Ni. J. Mater Sci. 52, 30 (2017) .

[57] Zhou X., Marchand D., McDowell DL., Zhu T., Song J., Chemomechanical Origin of Hydrogen Trapping at Grain Boundaries in fcc Metals. Physical review letters 116 (7), 075502 (2016).

[58] Zhou X., Song J., Effects of alloying elements on vacancies and vacancy-hydrogen clusters at coherent twin boundaries in nickel alloys. Acta Materialia 148 9-17 (2018).

[59] Sutton A.P. and Balluffi R.W. Interfaces in Crystalline Materials, Clarendon Press, Oxford, (1995).

[60] Priester L. Grain Boundaries From Theory to Engineering. Springer; New York (2013).

[61] Hallil A., Metsue A., Bouhattate J., Feaugas X., Correlation between vacancy formation and $\Sigma 3$ grain boundary structures in nickel from atomistic simulations. Phil. Mag. 96 2088-2114 (2016).

[62] Dingreville R., Berbenni S., On the interaction of solutes with grain boundaries. Acta Materialia 104, 237-249 (2016).

[63] Du Y.A., Ismer L., Rogal J., Hickel T., Neugebauer J. and Drautz R., First-principles study on the interaction of $\mathrm{H}$ interstitials with grain boundaries in $\alpha$ - and $\gamma$-Fe. Physical Review B 84, 144121 (2011).

[64] Zhou, X., Mousseau, N., Song, J.Is, Hydrogen Diffusion along Grain Boundaries Fast or Slow? Atomistic Origin and Mechanistic Modeling Physical Review Letters, 122 (21), art. no. 215501(2019).

[65] Olmsted, D.L., Foiles S.M., Holm E.A., Survey of computed grain boundary properties in facecentered cubic metals: I. Grain boundary energy, Acta Materialia. 57, 3694-3703 (2009).

[66] Lejcek P., Grain Boundary Segregation in Metals, Springer Series in materials science (2010).

[67] Priester L., Grain Boundaries: From Theory to Engineering, Springer Series in materials science (2013).

[68] Kirchheim, R. \& Hirth, J. P. Stress and solubility for solutes with asymmetrical distortion fields. Acta metall. 35, 2899-2903 (1987).

[69] Dyre J.C., Dominance of shear elastic energy far from a point defect in a solid, Phys. Rev. B 75. 
092102 (2007).

[70] Taketomi S., Matsumoto R., Miyazaki N., Atomistic study of hydrogen distribution and diffusion around a $\{112\}<111>$ edge dislocation in alpha iron. Acta Materialia 56 3761-3769 (2008).

[71] Matsumoto R., Inoue Y., Taketomi S., Miyazaki N., Influence of shear strain on the hydrogen trapped in bcc-Fe: A first-principles-based study. Scripta Material 60 555-558 (2009).

[72] Dudarev S.L., Ma P.W. Elastic fields, dipole tensors and interaction between self-interstitial atom defects in transition metals. Phys. Rev. Mater. 2033602 (2018).

[73] Hachet G., Li J., Hallil A.M., Metsue A., Oudriss A., Bouhattate J., Feaugas X., A multi-scale analysis of the different interactions between defects $\mathrm{T}$ and hydrogen: A review on the contribution of the elastic fields, Engineering Fracture Mechanics, 21810662 (2019).

[74] Nazarov R., Majevadia J. S., Patel M., Wenman M. R., Balint D. S., Neugebauer J., and Sutton A. P. First-principles calculation of the elastic dipole tensor of a point defect: Application to hydrogen in $\alpha$-zirconium. Phys Rev B 94241112 (2016).

[75] Clouet E., Varvenne C., Jourdan T. Elastic modeling of point-defects and their interaction. Comp. Mater. Science 147 49-63 (2018).

[76] Eshelby J.D., The continuum theory of lattice defects, Sol. State Phys. 379 (1956).

[77] Mura T., Micromechanics of defects in solids, Martinus Nijhoff Publishers, (1987).

[78] Sofronis P., Birnbaum H.K.. Mechanics of the hydrogen-dislocation-impurity interactions-I. increasing shear modulus. Journal of the Mechanics and Physics of Solids. 43(1), 49-90 (1995).

[79] Chateau J.P., Delafosse D., and Magnin T.. Numerical simulations of hydrogen-dislocation interactions in FCC stainless steels.: part I: Hydrogen-dislocation interactions in bulk crystals. Acta Materialia. 50(6)1507-1522 (2002).

[80] Delafosse D., Hydrogen effects on the plasticity of face centred cubic (fcc) crystals, in: R.D. Gangloff, B.P. Somerday (Eds.), Gaseous Hydrogen Embrit- tlement of Materials in Energy Technologies: Mechanisms, Modelling and Future Developments, vol. 2, Woodhead Publishing LtD $247 \mathrm{e} 285$ (2002).

[81] Cai W., Sills R., Barnett D., Nix W. Modeling a distribution of point defects as misfitting inclusions in stressed solids. J. Mech. Phys. Solid. 66, 154e171 (2014).

[82] Metsue, A., Oudriss, A., Feaugas, X., "Displacement field induced by a vacancy in nickel and some implications for the solubility of hydrogen”, Philosophical Magazine, 94 (34), 3978-3991 (2014).

[83] Wimmer E, Wolf W, Sticht J, Saxe P, Geller C, Najafabadi R, et al. Temperature-dependent diffusion coefficients from ab initio computations: Hydrogen in nickel. Phys Rev B. 77, 134305 (2008).

[84] Metsue A., Oudriss A., Feaugas X., Trapping/detrapping kinetic rates of hydrogen around a vacancy in nickel and some consequences on the hydrogen-vacancy clusters thermodynamic equilibrium; Computational Materials Sciences 151, 144-152 (2018)

[85] Larché, F. C., Cahn, J. W. The effect of self-stress in solids. Acta metall. 32, 1835-1845 (1982).

[86] Kirchheim R., Hirth, J. P. Stress and solubility for solutes with asymmetrical distortion fields. Acta metall. 35, 2899-2903 (1985).

[87] Vattré A., Jourdan T., Ding H., Marinica M.C., Demkowicz M.J.. Non-random walk diffusion enhances the sink strength of semicoherent interfaces. Nature Com. 710424 (2016).

[88] McLean D. Grain boundaries in metals. Monographs on the physics and chemistry of materials. Clarendon press (1957).

[89] Huber L., Hadian R., Grabowski B.,Neugebauer J. A machine learning approach to model solute grain boundary segregation. npj Comput Mater 4, 64 (2018).

[90] White, C., Coghlan, W. The spectrum of binding energies approach to grain boundary segregation. Metall. Mater. Trans. A 8, 1403-1412 (1977).

[91] von Pezold J., Lymperakis L., Neugebeauer J. Hydrogen-enhanced local plasticity at dilute bulk H concentrations: The role of $\mathrm{H}-\mathrm{H}$ interactions and the formation of local hydrides. Acta Materialia 59, Issue 8 2969-2980 (2011).

[92] Song J., Curtin W.A. A nanoscale mechanism of hydrogen embrittlement in metals. Acta Materialia 59 1557-1569 (2011).

[93] Lezzar B., Khalfallah O., Larere A., Paidar V., Hardouin Duparc O. Detailed analysis of the segregation driving forces for $\mathrm{Ni}(\mathrm{Ag})$ and $\mathrm{Ag}(\mathrm{Ni})$ in the $\Sigma=11\{113\}$ and $\Sigma=11\{332\}$ grain boundaries. Acta Materialia 52, Issue 9, 2809-2818 (2004).

[94] Juhas M. C., Priester L., Biscondi M. Correlation between geometry and chemistry of a near $\Sigma 11$ grain boundary. Mater. Science and Eng., A185 71-76 (1994).

[95] Feaugas, X., Haddou, H. Grain-size effects on tensile behavior of nickel and AISI 316L stainless steel. Metall Mater Trans A 34 2329-2340 (2003).

[96] Godon A., Creus J., Feaugas X., Conforto E., Pichon L., Armand C., Savall C. Characterization of electrodeposited nickel coatings from sulphamate electrolyte without additive. Materials 
Characterization. 62, Issue 2 164-173 (2011).

[97] Oudriss, A., Feaugas, X. Length scales and scaling laws for dislocation cells developed during monotonic deformation of (001) nickel single crystal. Int. J. Plast. 78, 187-202 (2016).

[98] Foiles S.M., Baskes M.I., Melius C.F., Daw M.S., Calculation of hydrogen dissociation pathways on nickel using the embedded atom method. J. Less Common Metals 130 465-473 (1987).

[99] Devanathan, M. A. V., Stachurski, Z. The adsorption and diffusion of electrolytic hydrogen in palladium. Proc. R. Soc. A. 170, 1340 (1962).

[100] Jónsson H., Mills G., Jacobsen K.W., Nudged elastic band method for finding minimum energy paths of transitions, in: Class. Quantum Dyn. Condens. Phase Simul., World Scientific, 385-404 (1998).

[101] Henkelman G., Uberuaga B.P., Jónsson H., A climbing image nudged elastic band method for finding saddle points and minimum energy paths, J. Chem. Phys. 113 9901-9904 (2000).

[102] Traisnel C., Metsue A., Oudriss A., Bouhattate J., Feaugas X. About the crystallographic orientation impact on hydrogen solubility and diffusivity near surface of nickel single crystals: a connection between long range and short-range energies, Computational Mater. Science, to be published (2020).

\section{Acknowledgements}

The authors would like to acknowledge our colleagues E. Conforto, R. Milet and C. Berziou for their contributions to the TEM and hydrogen charging facilities in the LaSIE Laboratory. This work is supported by the French National Research Agency (ANR) through Grant ANR-13-JS090015 CRISTALHYD.

\section{Author contributions}

J. Bouhattate, A. Oudriss, A. Metsue and X. Feaugas defined the selection of grain boundaries. J. $\mathrm{Li}, \mathrm{A}$. Oudriss and X. Feaugas characterized the grain boundaries using TEM and SEM/EBSD and the hydrogen states based on TDS measurements. J. Li and M. Hallil conducted the atomistic calculation. X. Feaugas, A. Metsue,A. Oudriss and J. Bouhattate carried out the elastic calculation of stress field and elastic energy and manage this work. All the authors contribute to data interpretation and to manuscript preparation.

\section{Impact Statement}

We clearly established the impact of the geometry and the deformation of hydrogen insertion site on the diffusion path and segregation process along grain-boundaries. The analyses offer the opportunity to clearly precise the trapping process on grain-boundary. A well-defined effect of deviatoric and hydrostatic elastic energies on segregation energy was identified at a short-range scale and additionally significant impact of a long-range elastic distortion observed for some GBs configurations.

\section{Keywords}

Nickel, hydrogen, grain-boundaries, excess volume, segregation energy, diffusion path, elastic dilatational and distortion energies.

\section{Competing Interests statement}

The authors declare no competing interests. 
Figures
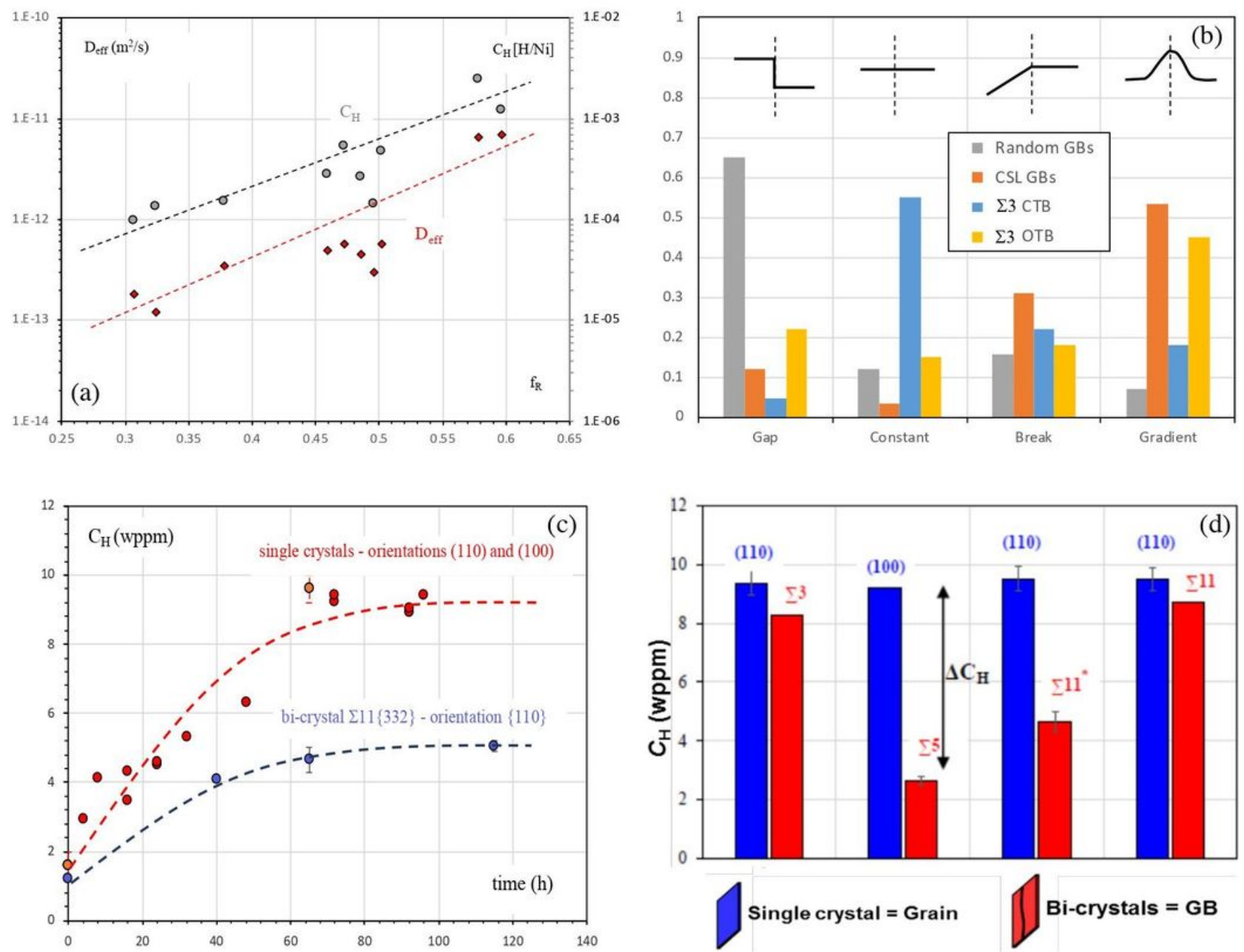

Figure 1

Please see the Manuscript PDF file for the complete figure caption 

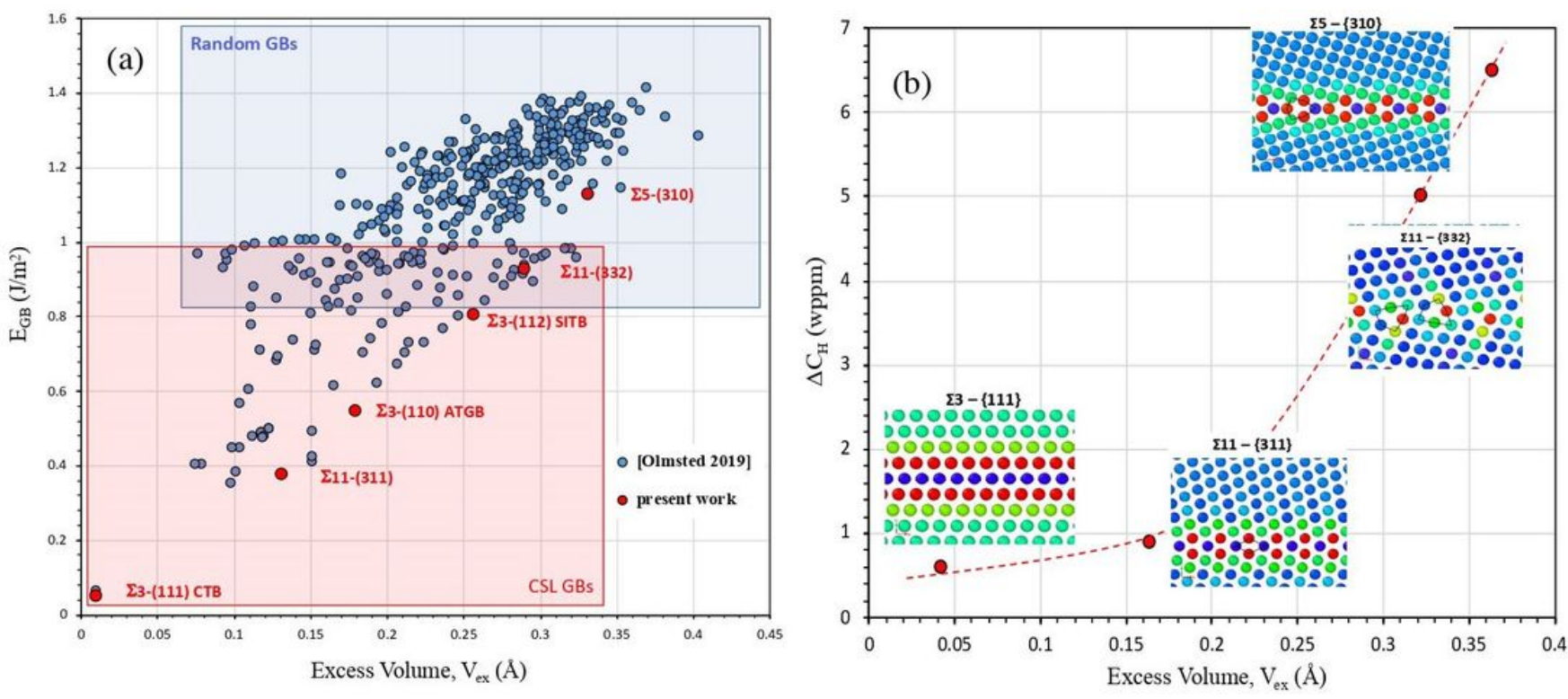

Figure 2

Please see the Manuscript PDF file for the complete figure caption 

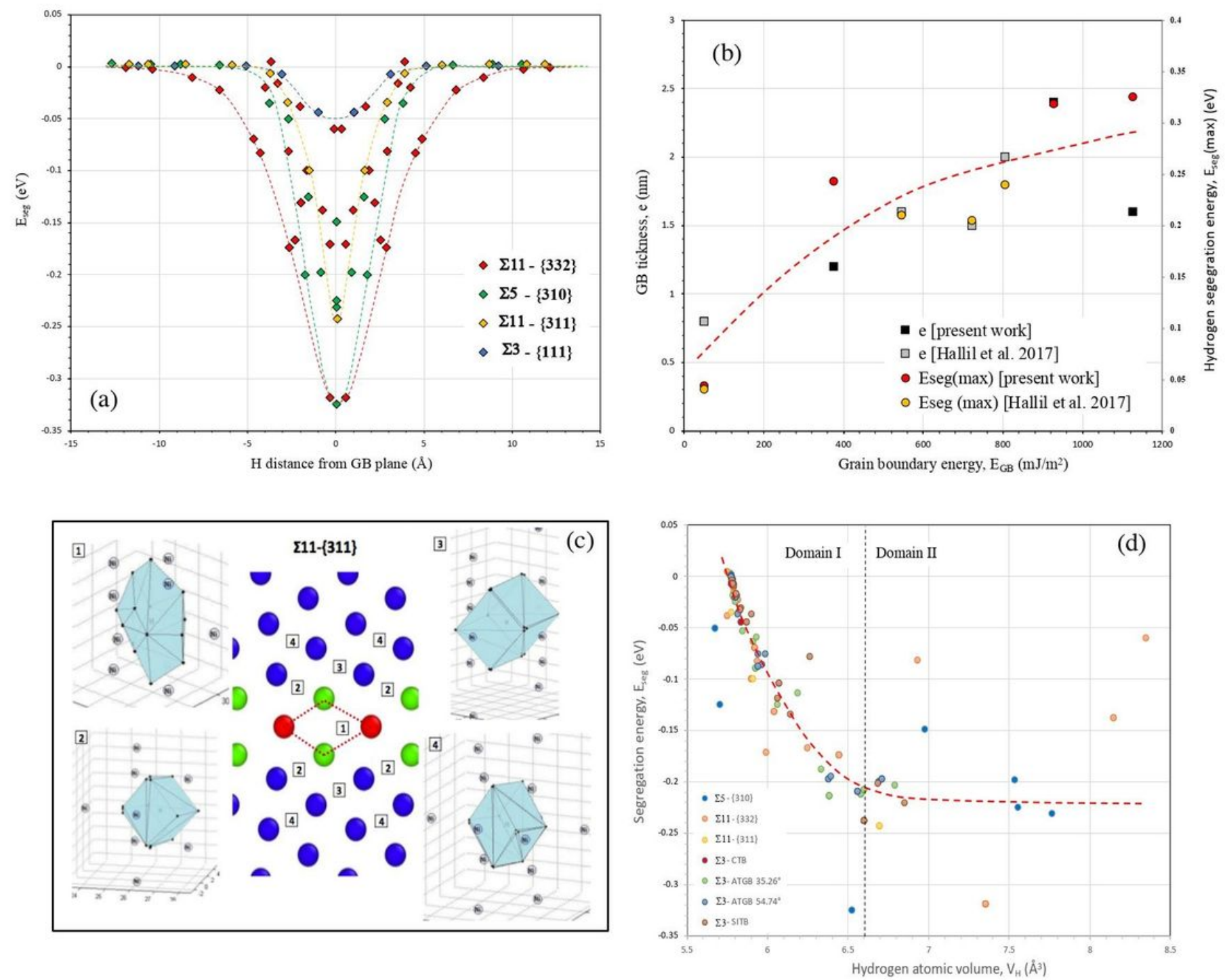

Figure 3

Please see the Manuscript PDF file for the complete figure caption 

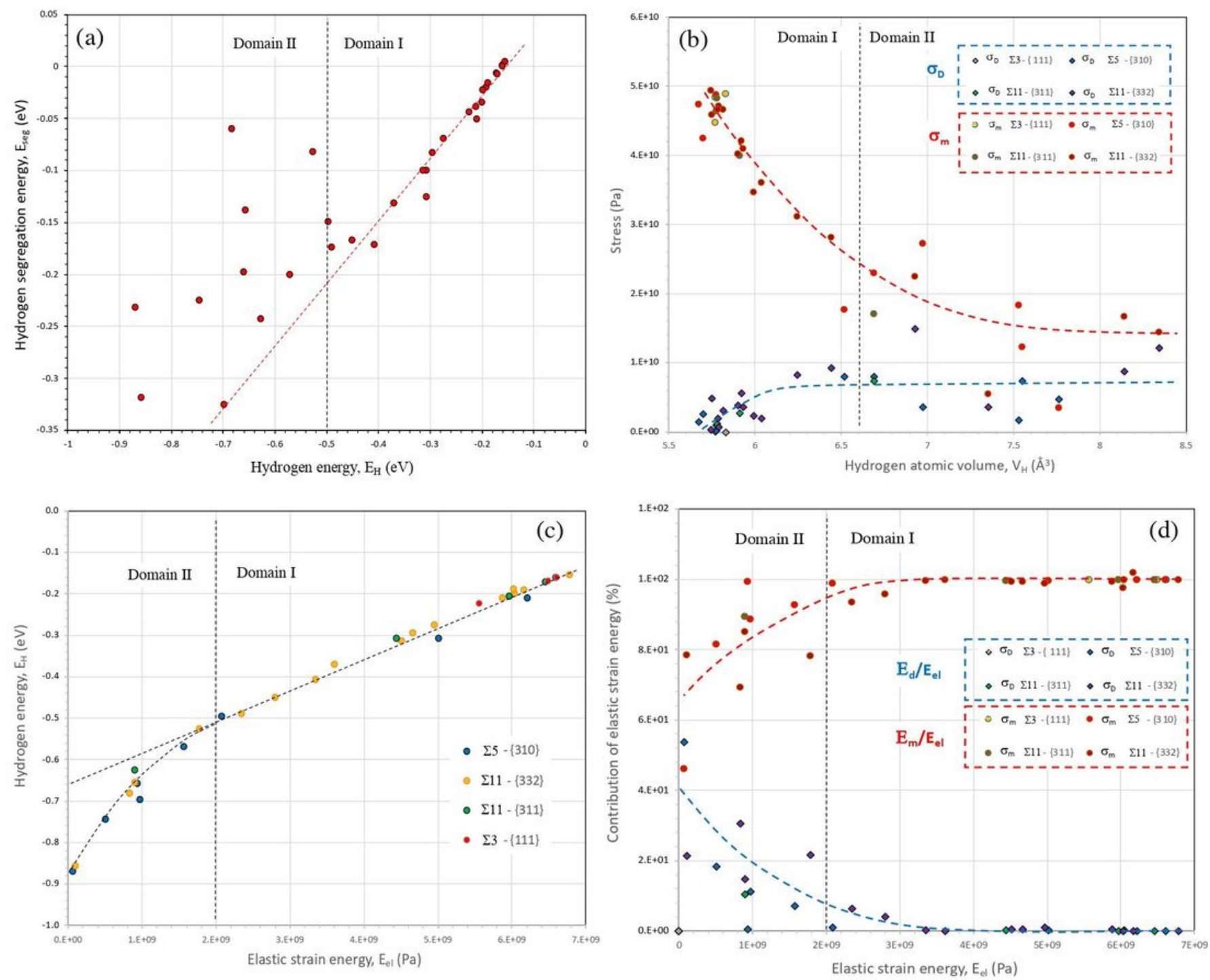

Figure 4

Please see the Manuscript PDF file for the complete figure caption 


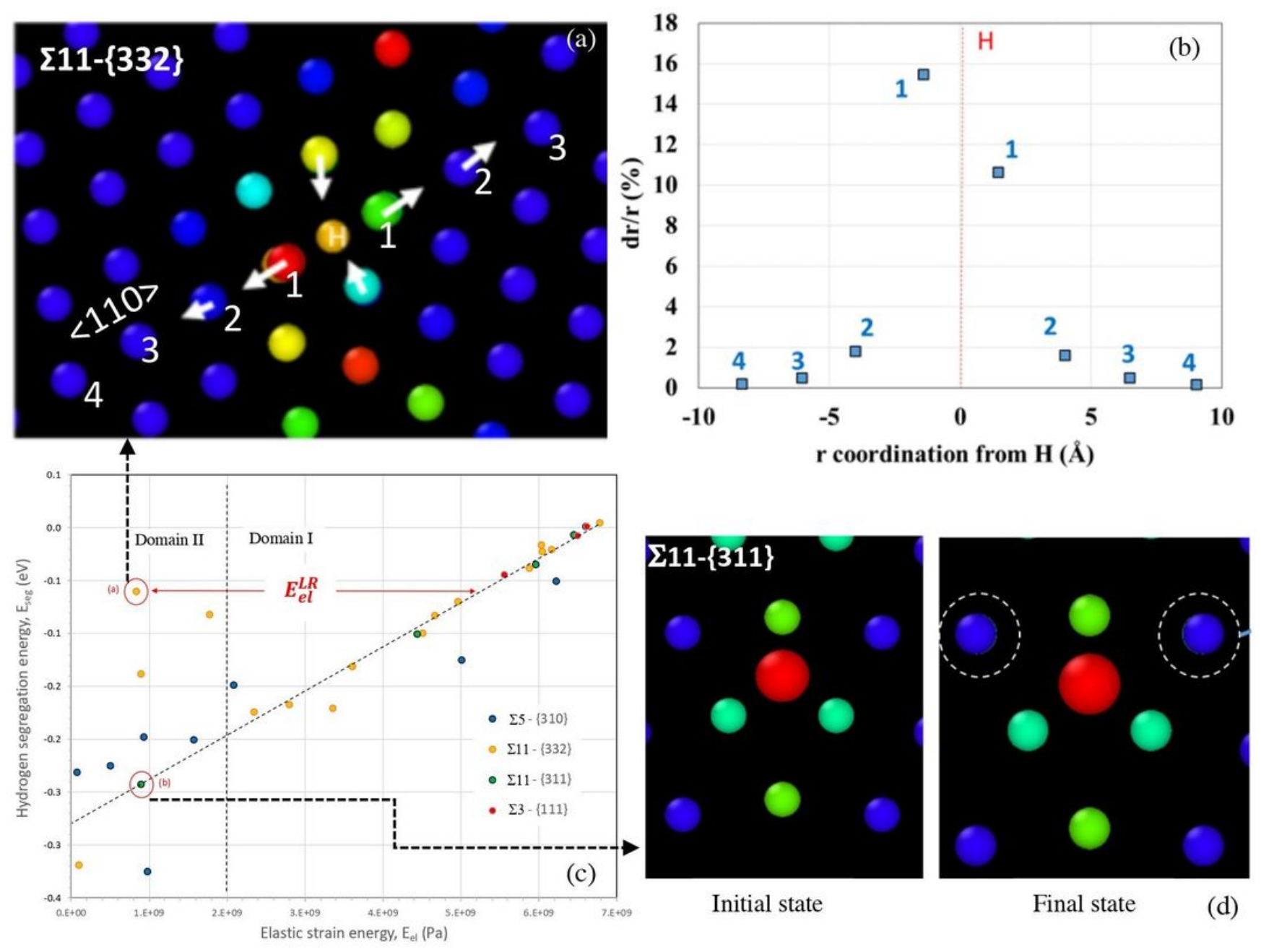

Figure 5

Please see the Manuscript PDF file for the complete figure caption 
(a)

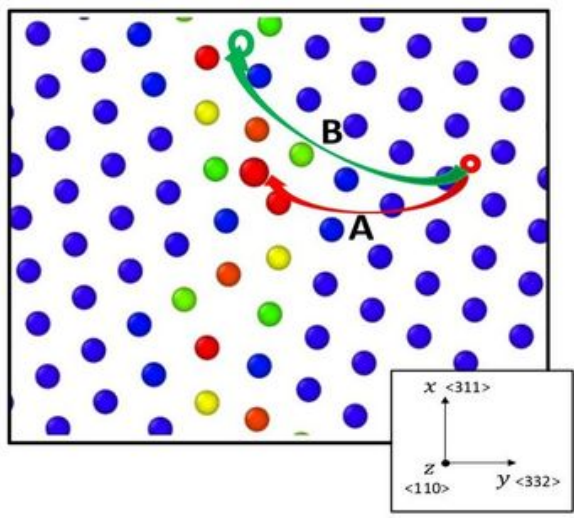

(b)

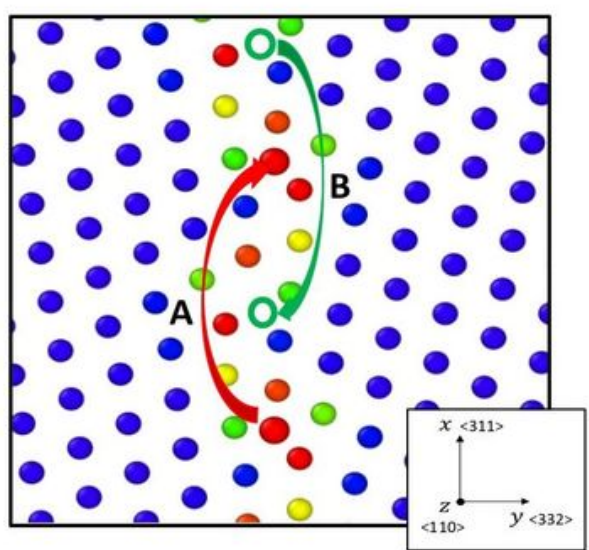

(c)

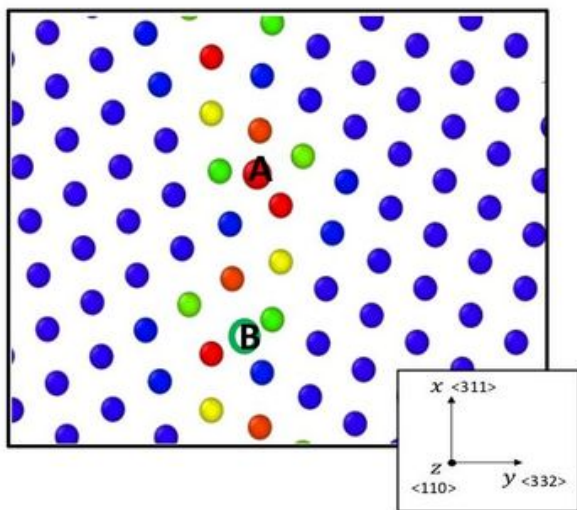

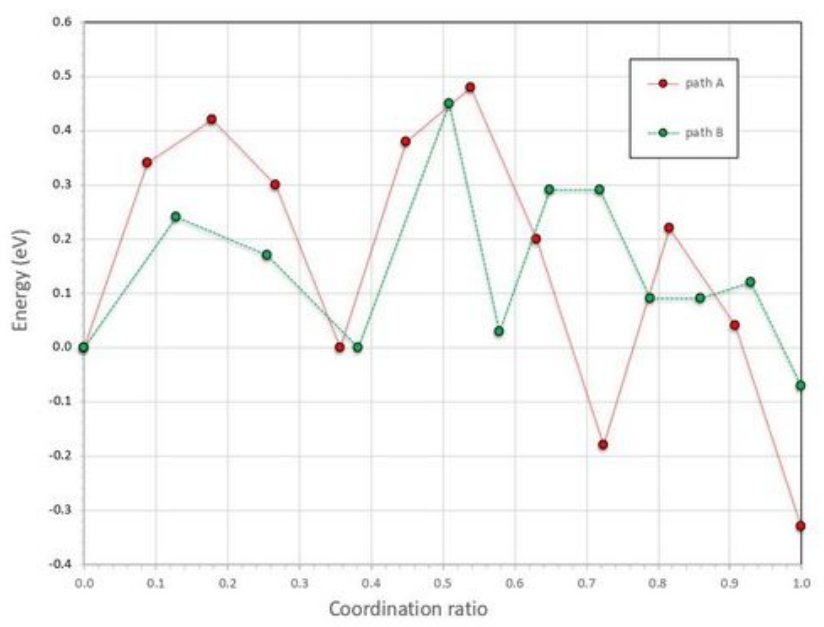
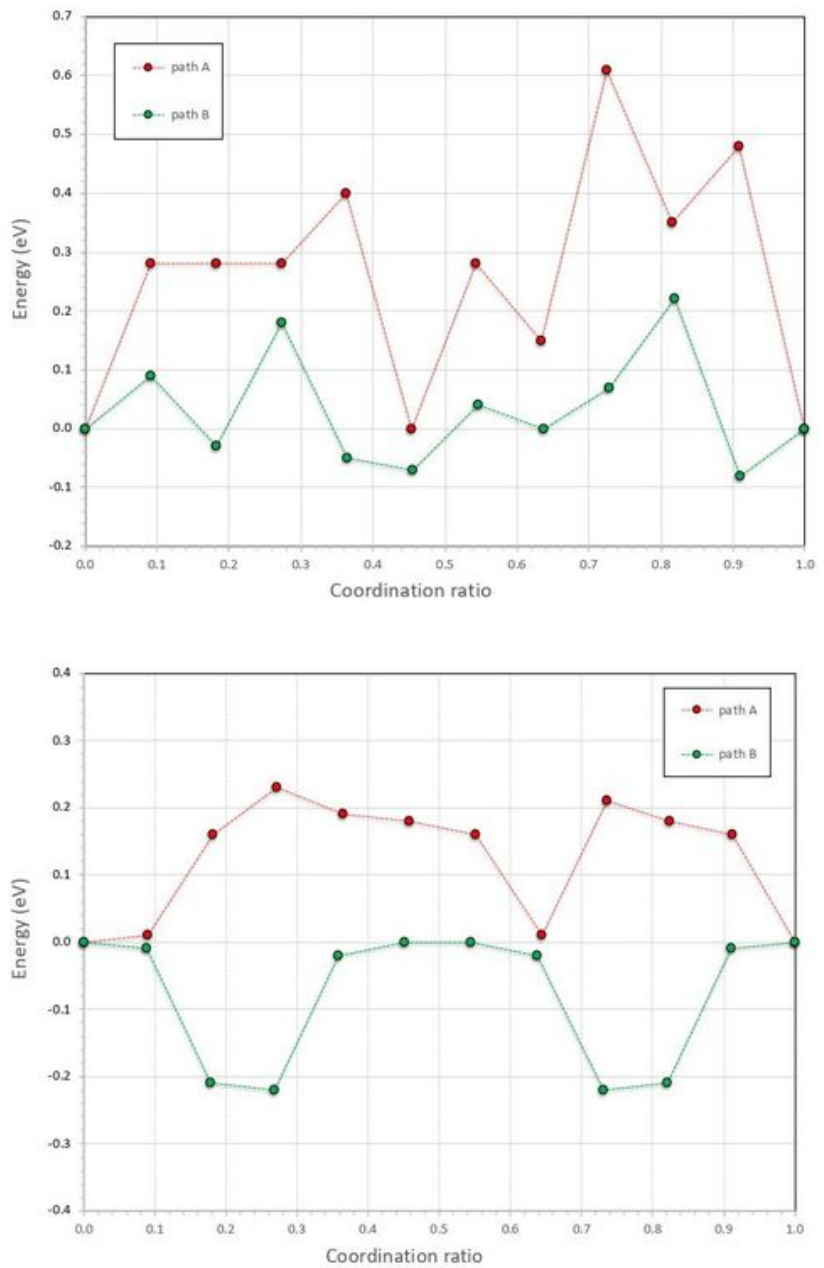

Figure 6

Please see the Manuscript PDF file for the complete figure caption 
(a)
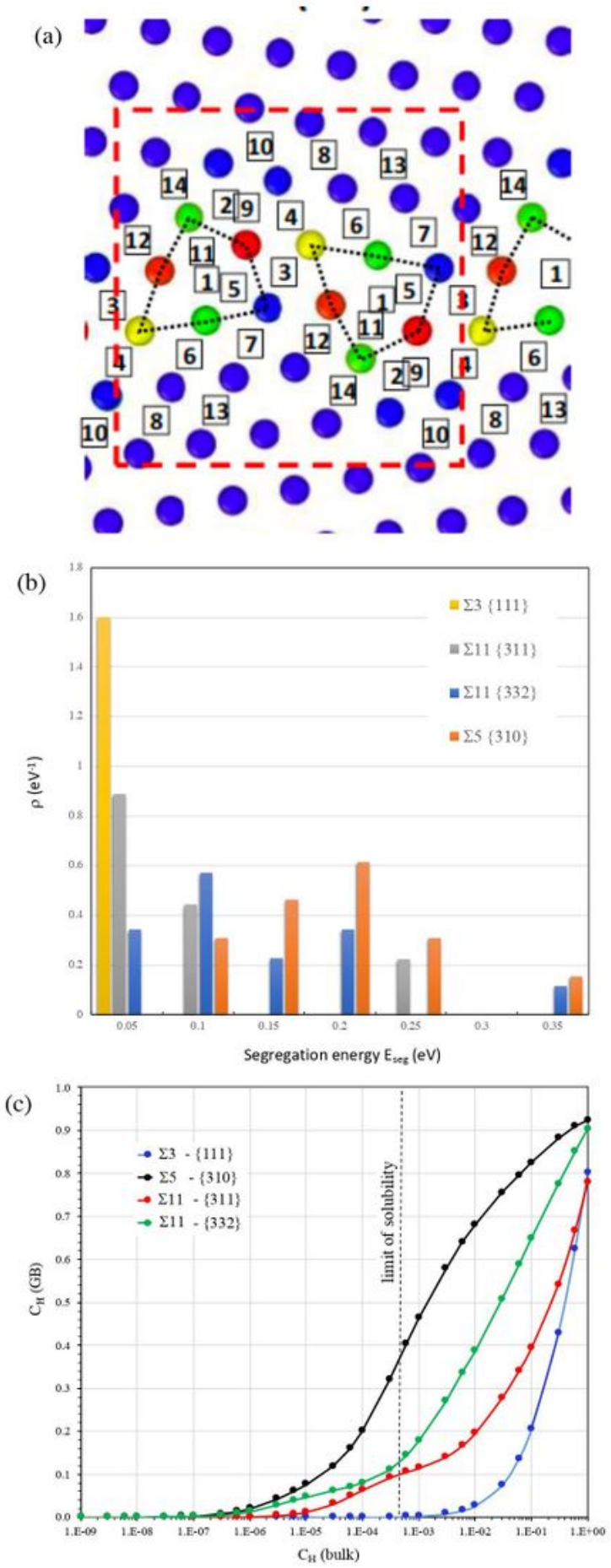

Figure 7

Please see the Manuscript PDF file for the complete figure caption 


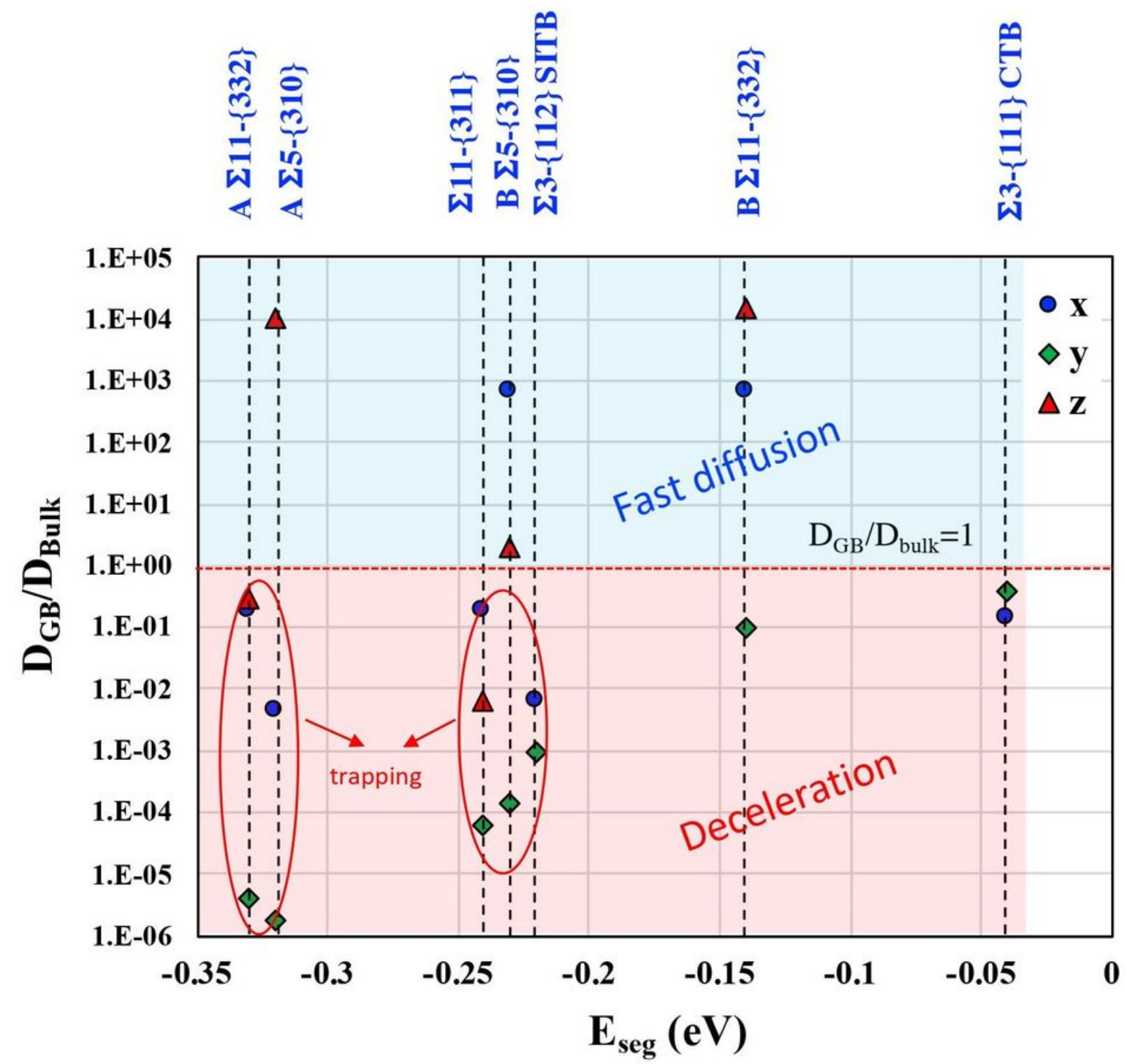

Figure 8

Please see the Manuscript PDF file for the complete figure caption

\section{Supplementary Files}

This is a list of supplementary files associated with this preprint. Click to download.

- A1.jpg 